\title{
Analytical theory of short-pulse free-electron laser oscillators
}

\author{
N. Piovella, ${ }^{1}$ P. Chaix,${ }^{1}$ G. Shvets,${ }^{2}$ and D. A. Jaroszynski ${ }^{1}$ \\ ${ }^{1}$ Commissariat à l'Energie Atomique, Service de Physique et Techniques Nucléaires, \\ Boîte Postale 12, 91680 Bruyères-le-Châtel, France \\ ${ }^{2}$ Princeton Plasma Physics Laboratory, Princeton, New Jersey 08543
}

(Received 7 July 1995)

\begin{abstract}
A simple model for the nonlinear evolution of a short-pulse free-electron laser oscillator in the small gain regime is derived. An analysis of the linearized system allows the definition and calculation of the eigenmodes characterizing the small signal regime. An arbitrary solution of the nonlinear system can then be expanded in terms of these supermodes. In the single-supermode approximation, the system reduces to a Landau-Ginzburg equation, which allows the efficiency and saturated power to be obtained as functions of cavity detuning and cavity losses. In the limit of small cavity detuning, electrons emit superradiantly, with an efficiency inversely proportional to the number of radiation wavelengths within the optical pulse, and power proportional to the square of the bunch charge. In the multisupermode regime, limit cycles and period doubling behavior are observed and interpreted as a competition between supermodes. Finally, the analytical and numerical results are compared with the experimental observations from the Free-Electron Laser for Infrared eXperiments experiment.
\end{abstract}

PACS number(s): 41.60.Cr, 42.60.Jf

\section{INTRODUCTION}

In this paper we study the linear and nonlinear evolution of radiation pulses in a small-gain free-electron laser (FEL) driven by electron pulses much shorter than the slippage length, $\Delta=\lambda N_{w}$, where $N_{w}$ is the number of undulator periods and $\lambda$ is the radiation wavelength [1]. We derive a system of equations for the radiation amplitude and the collective electron variables describing the multipass evolution in the cavity. The system is controlled by only two independent parameters, cavity detuning and losses. In the small signal regime eigenvalues and eigenmodes (supermodes) of the linearized system are found as functions of the cavity detuning. This allows one to calculate gain, linear frequency shift, and optical power for different supermodes. The maximum gain per pass,

$$
\mathcal{G}_{\text {opt }}=0.1875\left(L_{b} / \Delta\right) g_{0},
$$

occurs for $\delta \mathcal{L}_{\text {opt }}=0.0225 L_{b} g_{0}$, where $\delta \mathcal{L}$ is the cavity shortening, $L_{b}$ is the electron beam length, and $g_{0}[2]$ is the usual $\mathrm{cw}$ small gain parameter.

The supermodes are then used to describe saturation, expanding the complete solution of the nonlinear system in terms of the eigenfunctions of the linear problem. In the single supermode operation, the model reduces to a Landau-Ginzburg equation describing steady-state saturation. This allows one to calculate efficiency and saturated power as a function of cavity detuning and losses. For small losses, the efficiency is maximum when the cavity length is close to that for vacuum synchronism. An approximate evaluation of the supermode at small cavity detuning shows analytically that at the max- imum efficiency operation point the emission is superradiant. Maximum efficiency occurs approximately for $\delta \mathcal{L} \sim 0.1815 L_{b}\left(\alpha_{0} L_{c} / L_{b}\right)^{3 / 2}$, where $\alpha_{0}$ is the total cavity loss, $L_{c}=\lambda / 4 \pi \rho$, the cooperation length [3], $\rho \propto I_{b}^{1 / 3}$, the fundamental FEL parameter [4] and $I_{b}$, the peak electron current. For a cavity length corresponding to optimum efficiency, the gain is $\mathcal{G} \sim 5 \alpha_{0} / 3$ and the optical pulse is a narrow spike of $N_{s}=\left(L_{c} / 2 \lambda\right) \sqrt{\alpha_{0} L_{c} / L_{b}} \ll N_{w}$ optical periods with a peak power scaling as the square of the beam charge,

$$
P_{s} \sim \rho P_{e}\left(\frac{L_{b}}{\alpha_{0} L_{c}}\right)^{2},
$$

where $P_{e}$ is the electron beam power, and the efficiency is

$$
\eta \sim \frac{1}{8 \pi N_{s}}=\rho \sqrt{\frac{L_{b}}{\alpha_{0} L_{c}}}
$$

Although short-pulse effects reduce the gain from the $\mathrm{cw}$ value $\mathcal{G}=0.27 g_{0}$ [2], efficiency is larger than the cw value $\eta \sim 1 / 2 N_{w}$.

For small values of both cavity detuning and losses, different supermodes can be nonlinearly excited, allowing for a multimode regime where the optical power oscillates periodically over several hundred passes $[5,6]$. We investigate numerically the transition from the quasi-steadystate regime, where one supermode dominates, to a limitcycle oscillation regime, where at least two supermodes have comparable amplitudes. As cavity detuning or cavity loss decrease, the equilibrium solution becomes unstable and period-doubling cascade leads to chaotic behavior [7]. In the case of chaotic behavior the number 
of supermodes involved in the dynamics becomes larger than two. Various regimes of nonlinear operation of the free-electron laser oscillator, such as stable point, limitcycle oscillation and period doubling are investigated in terms of supermodes. An accurate quantitative prediction of the nonlinear behavior requires the multielectron equations [see Eqs. (11) and (12)]. The objective of the present work, in which we use the reduced model [see Eqs. (16)-(20)], is to capture the essential nonlinear features of short-pulse FEL oscillators.

\section{THE BASIC MODEL}

We start with the standard set of FEL equations, derived in the Compton approximation and recast in a dimensionless form, for the complex field amplitude $A$ and the electron phase and energy, $\theta$ and $p[3]$ :

$$
\begin{aligned}
& \frac{\partial A(u, \xi)}{\partial u}=g_{0} \chi(u)\langle\exp [-i \theta(u, \xi)]\rangle \\
& \frac{\partial \theta(u, \xi)}{\partial \xi}=p(u, \xi) \\
& \frac{\partial p(u, \xi)}{\partial \xi}=-\{A(u, \xi) \exp [i \theta(u, \xi)]+\text { c.c. }\}
\end{aligned}
$$

In these equations, $u=\left(z-v_{\|} t\right) / \beta_{\|} \Delta, \xi=(c t-z) / \Delta$, with $u+\xi=1$, where $z$ is the distance along the axis of an undulator of length $L_{w}=\lambda_{w} N_{w}$, period $\lambda_{w}=2 \pi / k_{w}$, and rms parameter $a_{w}, \lambda=\lambda_{w}\left(1-\beta_{\|}\right) / \beta_{\|} \simeq \lambda_{w}(1+$ $\left.a_{w}^{2}\right) / 2 \gamma_{0}^{2}$ is the resonant wavelength, and $v_{\|}=c \beta_{\|}$is the average longitudinal beam velocity; the other variables are the electron phase $\theta=\left(k+k_{w}\right) z-c k t$, with $k=2 \pi / \lambda$, the electron energy detuning, $p=4 \pi N_{w}\left(\gamma-\gamma_{0}\right) / \gamma_{0}$, the slowly varying complex amplitude of the radiation field $A$, defined such that $|A|^{2}=4 \pi N_{w} g_{0} P(z, t) / P_{e}$ where $P(z, t)$ is the intracavity optical power, $P_{e}=$ $m c^{2} \gamma_{0}\left(I_{b} / e\right)$ is the electron beam power, $I_{b}$ is the peak electron current, and $m c^{2} \gamma_{0}$ is the initial beam energy; $g_{0}=4 \pi\left(N_{w} / \gamma_{0}\right)^{3}\left(I_{b} f / I_{0}\right)\left(a_{w} \lambda_{w} F / r_{b}\right)^{2}$ is the cw smallgain parameter [2], $F$ is 1 for a helical undulator and equal to the well-known difference of Bessel functions for a linear undulator, $r_{b}$ is the beam radius, $I_{0}=$ $4 \pi \epsilon_{0} m c^{3} / e \sim 17000 \mathrm{~A}$ is the Alfven limit current, and $f$ is the filling factor describing the transverse overlap between the optical and electron pulses. The parameter $g_{0}$ is related to the fundamental FEL parameter $\rho$ of Ref. [4], which is independent of the undulator length and scales as $I_{b}^{1 / 3}$, by $g_{0}=\left(4 \pi \rho N_{w}\right)^{3}$. In Eq. (4) the angular brackets indicate an average over the particles and the electron current profile is modeled as $I_{b}(u)=I_{b} \chi(u)$, with $\int d u \chi(u)=L_{b} / \Delta$, where $L_{b}$ is the mean length of the electron micropulse, defined as the charge divided by $I_{b} / c$, which is independent of the bunch shape.

In an oscillator, the radiation is reflected backward after amplification and then forward for the next pass through the undulator, so that the input field for the $(n+1)$ th pass is

$$
A_{0}^{(n+1)}(\xi-\delta)=r A_{f}^{(n)}(\xi)
$$

where $A_{f}$ and $A_{0}$ are, respectively, the fields at the end and at the entrance of the undulator, $r$ accounts for the reduction in amplitude due to energy losses at the mirrors, and $\delta=2 \delta \mathcal{L} / \Delta$ is the normalized cavity detuning, where $\delta \mathcal{L}$ is the cavity shortening relative to that for perfect synchronism between the cavity round-trip time for vacuum speed of light and the injection period of the electron micropulses. By shortening the cavity by $\delta \mathcal{L}$, the optical pulse is pushed forward in $\xi$ by $\delta$ on each pass .

In the $(u, \xi)$ plane, the undulator lies between the lines $u+\xi=0$ and $u+\xi=1$. The electrons move on lines of constant $u$ and the radiation, propagating in the same direction as the beam, moves on lines of constant $\xi$. The electrons and the radiation interact only when their trajectories in the $(u, \xi)$ plane intersect between the lines $u+\xi=0$ and $u+\xi=1$. Integrating Eq. (4) along $u$ and using Eq. (7), the input field for the $(n+1)$ th pass is

$$
\begin{aligned}
A_{0}^{(n+1)}(\xi-\delta)= & r A_{0}^{(n)}(\xi) \\
& +r g_{0} \int_{-\xi}^{1-\xi} d u \chi(u)\langle\exp [-i \theta(u, \xi)]\rangle
\end{aligned}
$$

We limit the analysis to ultrashort electron micropulses, with $\chi(u)=\left(L_{b} / \Delta\right) \delta(u)$, where $\delta(u)$ is the Dirac delta function. We assume a micropulse much shorter than the slippage length $\left(L_{b} \ll \Delta\right)$, but long with respect to the radiation wavelength, such that the phase of the electrons entering the undulator is uncorrelated with that of the radiation pulse. Thus $\theta$ is initially distributed uniformly between 0 and $2 \pi$. The case of a micropulse shorter than a radiation wavelength has been considered by Pinhasi and Gover [8], and should not be confused with the shortmicropulse case discussed here.

The linear gain per pass is assumed small, $\left(L_{b} / \Delta\right) g_{0}<$ 1 , so that the substitution $A(u, \xi)=A_{0}(\xi)$ can be made in Eq. (6). In this limit, the cavity shortening $\delta$ and the cavity losses $1-r$ are assumed small, so that in Eq. (8) $r=1-\alpha_{0} / 2$, with $\alpha_{0} \ll 1$, and $A^{(n+1)}(\xi-\delta)$ may be approximated by the following Taylor series expansion.

$$
A_{0}^{(n+1)}(\xi-\delta) \simeq A_{0}^{(n)}(\xi)+\frac{\partial A_{0}^{(n)}(\xi)}{\partial n}-\delta \frac{\partial A_{0}^{(n)}(\xi)}{\partial \xi}
$$

where the pass number $n$ is considered as a coarse-grained variable. Defining $A(\xi, \tau)=A_{0}^{(n)}(\xi)$,

$$
\tau=\gamma n, \quad \nu=\frac{\delta}{\gamma}=\frac{2 \delta \mathcal{L}}{\Delta \gamma}, \quad \alpha=\frac{\alpha_{0}}{\gamma},
$$

in terms of the gain parameter $\gamma=\left(L_{b} / \Delta\right) g_{0}$, the difference equation (8), with Eq. (9), and Eqs. (5) and (6), give

$$
\begin{array}{r}
\frac{\partial A(\xi, \tau)}{\partial \tau}-\nu \frac{\partial A(\xi, \tau)}{\partial \xi}+\frac{\alpha}{2} A(\xi, \tau) \\
=\eta\langle\exp [-i \tilde{\theta}(\xi, \tau)]\rangle,
\end{array}
$$




$$
\frac{\partial^{2} \tilde{\theta}(\xi, \tau)}{\partial \xi^{2}}=-\{A(\xi, \tau) \exp [i \tilde{\theta}(\xi, \tau)]+\text { c.c. }\}
$$

where $\eta=1$ for $0<\xi<1$ and $\eta=0$ elsewhere, and $\tilde{\theta}(\xi, \tau)=\theta(u=0, \xi)$. The gain parameter $\gamma$ accounts for the reduction of the usual $\mathrm{cw}$ parameter $g_{0}$ due to short-pulse effects, by the factor $L_{b} / \Delta<1$.

As a result of the electron bunch being very short, the radiation interacts with all the electrons over the slippage length $0<\xi<1$. Due to the cavity shortening $\nu$, radiation moves forwards or backwards in $\xi$ during successive passes through the cavity, depending on whether $\nu$ is, respectively, negative or positive. In the first case (cavity longer than perfect synchronism), a boundary condition for $A$ must be assigned to the leading edge $\xi=0$, and the radiation is shifted by $|\nu| \tau$ along the positive direction of $\xi$, leaving the interaction region through the boundary $\xi=1$; in this case the radiation propagates in the same direction as the electrons. In the second case, when $\nu$ is positive (cavity shorter than perfect synchronism), a boundary condition for $A$ must be assigned to the trailing edge $\xi=1$, and the radiation propagates backwards, leaving the interaction region through the boundary $\xi=0$ and moving towards the electrons. The exact synchronism case $(\nu=0)$ has been studied in a previous work [9], showing that superradiant emission occurs in the limit of very low cavity loss. In the linear regime the radiation builds up with a nonexponential growth rate, with energy

$$
\mathcal{E}(\tau) \simeq\left[\mathcal{E}_{0} / 6 \pi \sqrt{3}(2 \tau)^{2 / 3}\right] \exp \left[\left(3 \sqrt{3} / 2^{2 / 3}\right) \tau^{1 / 3}-\alpha \tau\right],
$$

where $\mathcal{E}_{0}$ is the initial energy, and net gain (gain minus losses)

$$
\mathcal{G}(\tau)=\gamma\left[\frac{\sqrt{3}}{(2 \tau)^{2 / 3}}-\frac{2}{3 \tau}-\alpha\right] .
$$

If $\alpha \tau \gg 1$, the radiation decays after reaching the $\operatorname{maximum} \mathcal{E}_{\max } \sim\left(\mathcal{E}_{0} \alpha / 18 \pi\right) \exp \left[(3 \sqrt{3} / \alpha)^{1 / 2}\right]$ at $\tau \sim$ $(1 / 2)(\sqrt{3} / \alpha)^{3 / 2}$. If the losses are very small and the radiation grows to $\mathcal{E} \sim \pi^{4}$ (for example, for $\mathcal{E}_{0} \sim 10^{-4}$, $\alpha \sim 0.01$ and $\tau \sim 10^{3}$ ) the electrons perform a half synchrotron oscillation in phase and the emission is superradiant, with $A(\xi, \tau)=\tau A_{1}(\sqrt{\tau} \xi)$, where $A_{1}$ is the solution of the self-similar equations (14) and (15) of Ref. [9].

We observe that Eqs. (11) and (12) depend only on two parameters, scaled cavity detuning $\nu=2 \delta \mathcal{L} / \Delta \gamma$ and scaled cavity loss, $\alpha=\alpha_{0} / \gamma$.

Finally, it is worth noting that Eqs. (11) and (12) are similar to the ones derived for a backward wave oscillator (BWO) [10], where $\tau$ is the scaled time and $\xi$ is the scaled undulator length.

\section{THE REDUCED MODEL}

A description of the FEL dynamics in the nonlinear regime requires a numerical integration of $2 N+2$ cou- pled equations for the phases and the energies of $N \gg 1$ electrons and a complex field amplitude, Eqs. (11) and (12). It has been shown that the complete FEL dynamics can be approximately described in terms of few relevant collective variables [11], assuming the following truncation relation:

$$
\left\langle(\tilde{p}-\langle\tilde{p}\rangle)^{2} \exp (-i \tilde{\theta})\right\rangle=\left\langle(\tilde{p}-\langle\tilde{p}\rangle)^{2}\right\rangle\langle\exp (-i \tilde{\theta})\rangle,
$$

where $\tilde{p}=\partial \tilde{\theta} / \partial \xi$. Ansatz (15) results from a truncation, at the second order in the hierarchy, of equations for the cumulants of the phase-energy electron distribution. Introducing $B=\langle\exp (-i \tilde{\theta})\rangle, P=\langle\tilde{p} \exp (-i \tilde{\theta})\rangle, Q=\langle\tilde{p}\rangle$ and $S=\left\langle\tilde{p}^{2}\right\rangle$, Eqs. (11) and (12) reduce to the following set of closed equations [11]:

$$
\begin{aligned}
& \frac{\partial A}{\partial \tau}-\nu \frac{\partial A}{\partial \xi}+\frac{\alpha}{2} A=\eta B \\
& \frac{\partial B}{\partial \xi}=-i P \\
& \frac{\partial P}{\partial \xi}=-A-i S B-2 i Q P+2 i Q^{2} B \\
& \frac{\partial Q}{\partial \xi}=-\left[A B^{*}+\text { c.c. }\right] \\
& \frac{\partial S}{\partial \xi}=-2\left[A P^{*}+\text { c.c. }\right]
\end{aligned}
$$

where $\eta=1$ for $0<\xi<1$ and $\eta=0$ elsewhere. We will assume in the following a resonant monochromatic and unbunched initial beam, with $B=P=Q=S=0$ at $\xi=0$ and initial field $A(\xi, \tau=0)=A_{0}(\xi)$. It can be easily shown that the assumption of a nonresonant initial beam is equivalent to a constant frequency shift in the initial field. This frequency shift of the radiation in response to a nonresonant electron beam is established in the linear regime as discussed in [12].

It is worth remarking that the only "independent" variable of Eqs. (16)-(20) is the field amplitude $A$, governed by Eq. (16); indeed, for each value of $\tau$, the electron variables $B, P, Q, R$, and $S$ are determined as functions of $A$ by Eqs. (17)-(20). This results from the fact that in an oscillator the radiation is reflected back to the undulator entrance, where it begins interacting with freshly injected electrons.

We observe that Eqs. (16) and (19) yield an equation for the energy balance:

$$
\eta \frac{\partial Q}{\partial \xi}+\left(\frac{\partial}{\partial \tau}-\nu \frac{\partial}{\partial \xi}\right)|A|^{2}=-\alpha|A|^{2} .
$$

For $\nu>0$ we define the scaled radiation energy as

$$
\mathcal{E}(\tau)=\int_{-\nu \tau}^{1} d \xi|A(\xi, \tau)|^{2}
$$

Integration of Eq. (21) over $\xi$, and neglecting $A(\xi=$ $1, \tau$ ) (which is physically equivalent to a low spontaneous emission level), yields the efficiency as a function of the scaled pass number $\tau$ :

$$
\eta(\tau) \equiv-\frac{Q(\xi=1, \tau)}{4 \pi N_{w}}=\frac{1}{4 \pi N_{w}}\left(\frac{d}{d \tau}+\alpha\right) \mathcal{E}(\tau)
$$


Finally we also note that, for $\nu>0$, the field propagates freely in the region $-\nu \tau<\xi<0$, decaying at a rate given by the cavity loss $\alpha$ :

$$
A_{\xi<0}(\xi, \tau)=e^{\alpha \xi / 2 \nu} A(\xi=0, \tau+\xi / \nu) .
$$

\section{LINEAR REGIME}

The linear regime has been studied numerically for an arbitrary beam profile $\chi(u)$ in Ref. [13] and analytically in the long-bunch approximation, $L_{b} \gg \Delta$, in Refs. $[14,15]$. In our case, the small-signal regime $(A \ll 1)$ is described by the linearized equations, for $0<\xi<1$ :

$$
\begin{aligned}
& \frac{\partial A}{\partial \tau}-\nu \frac{\partial A}{\partial \xi}+\frac{\alpha}{2} A=B \\
& \frac{\partial B}{\partial \xi}=-i P \\
& \frac{\partial P}{\partial \xi}=-A
\end{aligned}
$$

We assume an initially unbunched beam, $B(\xi=0, \tau)=$ $P(\xi=0, \tau)=0$, and note that for perfect cavity synchronism $(\nu=0)$, no boundary condition on $A$ is required, because the radiation does not propagate along $\xi$. For $\nu<0$ or $\nu>0$ we assume $A(\xi=0, \tau)=A_{0}$ or $A(\xi=1, \tau)=A_{0}$, respectively, where $A_{0}$ characterizes the level of spontaneous emission that is amplified by the FEL interaction. In addition, the initial level of radiation in the oscillator cavity is assumed to match the level of spontaneous emission i.e., $A(\xi, \tau=0)=A_{0}$.

The solution of the set of linear equations (25)-(27) with inhomogeneous boundary conditions can be represented as a sum of time-independent solutions with inhomogeneous boundary conditions and a time-dependent solution with homogeneous boundary conditions. By assuming that the level of spontaneous emission $A_{0}$ is small, we will concentrate on the time-dependent solutions that can have a nonzero temporal growth rate and, hence, reach high amplitudes.

The eigenstates of Eqs. (25), (26), and (27) are solutions of the form

$$
\begin{aligned}
& A(\xi, \tau)=\exp [(\mu-\alpha / 2) \tau] \Phi(\xi) \\
& B(\xi, \tau)=\exp [(\mu-\alpha / 2) \tau] \Psi(\xi) \\
& P(\xi, \tau)=\exp [(\mu-\alpha / 2) \tau] \Gamma(\xi)
\end{aligned}
$$

where the steady-state gain per pass is $\mathcal{G}=2 \gamma \operatorname{Re} \mu$. Substituting (28)-(30) in Eqs. (25)-(27), we obtain

$$
\nu \Psi^{\prime \prime \prime}-\mu \Psi^{\prime \prime}+i \Psi=0
$$

and $\Psi^{\prime \prime}=i \Phi$, where a prime indicates a derivative with respect to $\xi$. From the boundary conditions on $B$ and $P, \Psi(0)=0$ and $\Psi^{\prime}(0)=0$; for $\nu<0$ or $\nu>0$ we assume $\Phi(0)=0$ or $\Phi(1)=0$, respectively. For $\nu \neq 0$, the solution

$$
\begin{aligned}
\Phi(\xi)= & \Phi_{N}\left[k_{1}^{2}\left(k_{2}-k_{3}\right) e^{-i k_{1} \xi}\right. \\
& \left.+k_{2}^{2}\left(k_{3}-k_{1}\right) e^{-i k_{2} \xi}+k_{3}^{2}\left(k_{1}-k_{2}\right) e^{-i k_{3} \xi}\right]
\end{aligned}
$$

where $\Phi_{N}$ is a normalization constant and $k_{j}$, with $j=$ $1,2,3$, are the complex roots of the cubic equation:

$$
\nu k^{3}-i \mu k^{2}+1=0 .
$$

We choose $\Phi$ normalized to 1 :

$$
\int_{0}^{1} d \xi|\Phi(\xi)|^{2}=1
$$

For $\nu<0, \Phi(0)=0$ and hence $\Phi(\xi)=0$, implying that no nonzero solutions exist for $\nu \leq 0$. This means that the time-independent solution is the solution of the set of Eqs. (25)-(27).

For $\nu>0$ the eigenvalue $\mu$ must satisfy the characteristic equation

$$
\begin{aligned}
& k_{1}^{2}\left(k_{2}-k_{3}\right) e^{-i k_{1}}+k_{2}^{2}\left(k_{3}-k_{1}\right) e^{-i k_{2}} \\
& +k_{3}^{2}\left(k_{1}-k_{2}\right) e^{-i k_{3}}=0 .
\end{aligned}
$$

Moreover, for $\xi<0$ the solution decays exponentially as $\Phi(\xi)=\Phi(0) \exp (\mu \xi / \nu)$ if $\mu$ has a positive real part. Eigenvalues $\mu$ can be obtained, as functions of the cavity detuning $\nu$, with a simple numerical iterative algorithm, searching the zero of Eq. (35). To facilitate the search, the threshold value of $\nu$, for which the eigenvalue $\mu$ has zero real part (i.e., zero gain), can be obtained first by a numerical scanning of only the imaginary part of $\mu$. Then, varying $\nu$ from the threshold, the eigenvalue can be then found by continuity. A discrete set of eigenvalues is found. No solutions with a positive real part exist for $\nu>0.13$, whereas the fundamental eigenvalue $\mu_{1}$ has a positive real part for $0<\nu<0.13$, with a maximum gain $\mathcal{G}_{1} / \gamma=0.1875$ at $\nu=0.045$; the second eigenvalue $\mu_{2}$ has a positive real part for $0<\nu<0.02$, with maximum gain $\mathcal{G}_{2} / \gamma=0.0412$ at $\nu=0.0048$; the third eigenvalue $\mu_{3}$ has a positive real part for $0<\nu<0.01$, with maximum gain $\mathcal{G}_{3} / \gamma=0.019$ at $\nu=0.0019$. In Fig. 1 we plot the scaled gain $\mathcal{G}_{n} / \gamma=2 \operatorname{Re} \mu_{n}$ [Fig. 1(a)] and the linear frequency shift $d \phi_{n} / d \tau=\operatorname{Im} \mu_{n}$ [Fig. 1(b)] for the first three eigenvalues $(n=1,2,3)$ as a function of the cavity detuning $\nu$.

For small values of $\nu$, the eigenvalues and the eigenfunctions can be approximated by the following expressions:

$$
\mu_{n} \simeq 3(\nu / 2)^{2 / 3}\left[e^{i \pi / 6}+n^{2} \pi^{2}(\nu / 2)^{2 / 3} e^{i 5 \pi / 6}\right]
$$

whereas the intensity is given by

$$
\left|\Phi_{n}(\xi)\right|^{2} \simeq\left(3 \sqrt{3} / n^{2} \pi^{2} \nu\right) \sin ^{2}(n \pi \xi) e^{\gamma_{n}(\xi-1)}
$$

and the associate phase change is given by

$$
\frac{d \psi_{n}}{d \xi}=(2 / \nu)^{1 / 3}\left[(1 / 2)+\left(n^{2} \pi^{2} / 3\right)(\nu / 2)^{2 / 3}\right],
$$

with $\gamma_{n}=\sqrt{3}(2 / \nu)^{1 / 3}\left[1-\left(2 n^{2} \pi^{2} / 3\right)(\nu / 2)^{2 / 3}\right]$ and $n=$ $1,2, \ldots$ From Eq. (36), the gain corresponding to the eigenvalue $\mu_{n}$ is 


$$
\mathcal{G}_{n}=3 \sqrt{3} \gamma(\nu / 2)^{2 / 3}\left[1-n^{2} \pi^{2}(\nu / 2)^{2 / 3}\right]
$$

and the linear frequency shift is

$$
\frac{d \phi_{n}}{d \tau}=\operatorname{Im} \mu_{n}=(3 / 2)(\nu / 2)^{2 / 3}\left[1+n^{2} \pi^{2}(\nu / 2)^{2 / 3}\right] .
$$

Hence, an increasing number of eigenvalues $\mu_{n}$ with comparable gain exists for small $\nu$, with maximum gain $\mathcal{G}_{n} \sim 3 \sqrt{3} \gamma / 4 n^{2} \pi^{2}$ at $\nu \sim 2 /(\sqrt{2} n \pi)^{3}$, tending toward the limit value for $\nu=0$ :

$$
\mathcal{G}=3 \sqrt{3} \gamma(\nu / 2)^{2 / 3}=3 \sqrt{3} \frac{L_{b}^{1 / 3}(\delta \mathcal{L})^{2 / 3}}{L_{c}}
$$

In Eq. (41) we have introduced a cooperation length, $L_{c}=\lambda / 4 \pi \rho[3]$. We note that for small cavity detuning the gain is independent of the number of undulator periods $N_{w}$ and proportional to the number of radiation wavelengths contained in the effective interaction length $L_{b}^{1 / 3}(\delta \mathcal{L})^{2 / 3}$. This can be explained by the fact that for very short cavity detuning the radiation pulse is much shorter than the slippage length, so that electrons interact with the radiation only over a fraction of the undulator proportional to the optical pulse width $[16,17]$. In this case the interaction length is not $\lambda N_{w}$ but $L_{b}^{1 / 3}(\delta \mathcal{L})^{2 / 3}$.
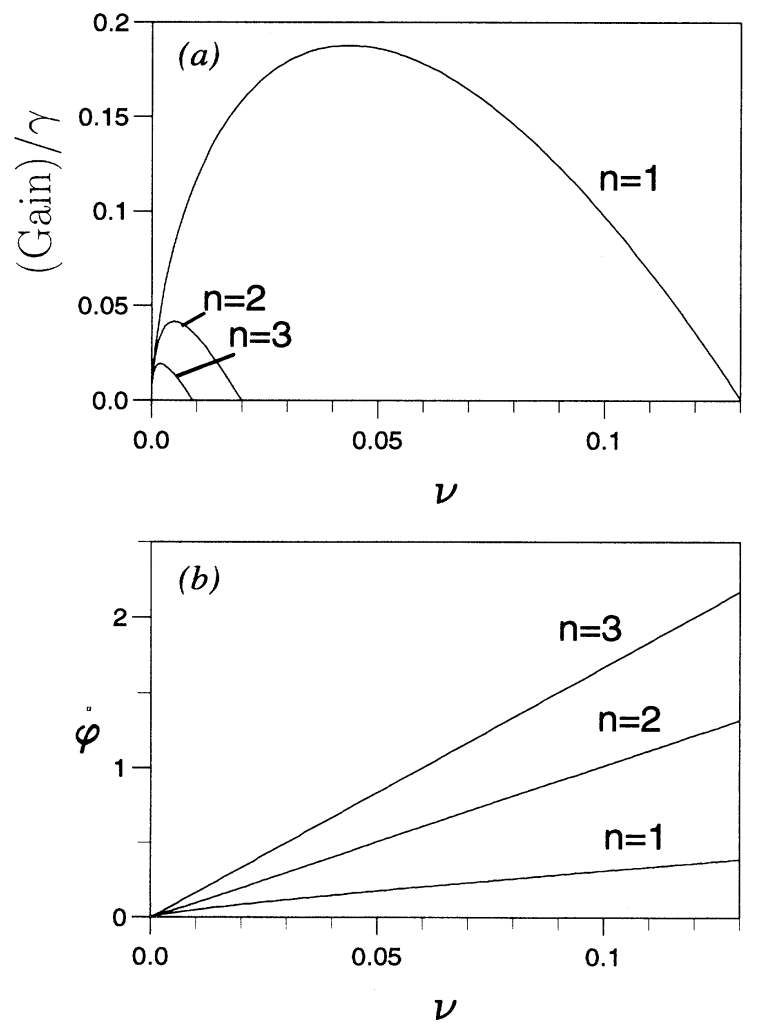

FIG. 1. (a) Linear gain, $\mathcal{G}_{n} / \gamma=2 \operatorname{Re} \mu_{n}$ and (b) linear phase shift, $\dot{\phi}_{n}=\operatorname{Im} \mu_{n}$ for the first three supermodes, $n=1,2,3$, vs cavity detuning $\nu=2 \delta \mathcal{L} / L_{b} g_{0}$.
It is interesting to observe that in the short cavity detuning limit, the gain is proportional to $I^{1 / 3}$, as in the linear stage of the superradiant regime $[18,19]$. This result for the linear gain suggests that the emission is superradiant for very small cavity shortening.

Figure 2 shows the fundamental eigenfunction $\left|\Phi_{1}\right|^{2}$ for $\nu=0.045$ (a) and $\nu=0.005$ (b); Fig. 3 shows $\left|\Phi_{2}\right|^{2}$ for $\nu=0.015$ (a) and $\nu=0.005(\mathrm{~b})$; and Fig. 4 shows $\left|\Phi_{3}\right|^{2}$ for $\nu=0.008$ (a) and $\nu=0.002$ (b). Using the notations of Ref. [13], we call a supermode the eigenmode $\Phi_{n}(\xi)$ corresponding to the eigenvalue $\mu_{n}$. From this analysis it follows that a single supermode, corresponding to the maximum gain $\mathcal{G}_{1}=2 \gamma \operatorname{Re} \mu_{1}$, is excited for $\nu \geq 0.02$. The second and third supermodes are excited for $\nu$ smaller than 0.02 and 0.01 , respectively. The linear phase drift $\dot{\phi}_{n}$ [Fig. 1(b)] increases with $\nu$ and is larger for increasing $n$.

Radiation pulse can be divided into two parts: the interacting part $0<\xi<1$, which participates in the FEL interaction process, and the decaying part $\xi<0$, which decays exponentially in $\xi$ due to cavity losses. For large cavity detuning, the interacting part of the radiation pulse has a broad profile, with a width comparable to the slippage length, whereas for small cavity detuning, the optical pulse peaks around the trailing edge $\xi=1$. In the former ( $\nu$ large), electrons interact with the radiation over the whole slippage length, whereas in the latter $(\nu$ small), the coupling with the radiation occurs only near the trailing edge $\xi=1$, reducing the effective interaction length and hence increasing the efficiency, as we will see in the next section. For small $\nu,\left|\Phi_{n}\right|^{2}$ is approximated by Eq. (37) and has $n+1$ nodes between $0 \leq \xi \leq 1$ and a sharp peak close to $\xi=1$. For very small $\nu$, the maximum $|\Phi|$ is $|\Phi|_{\max }^{2} \sim 12 \sqrt{3} / e^{2} \nu \gamma_{n}^{2} \sim\left(2 \sqrt{3} / e^{2}\right)(2 / \nu)^{1 / 3}$ at the position $\xi \sim 1-2 / \gamma_{n} \sim 1-(2 / \sqrt{3})(\nu / 2)^{1 / 3}$ with a width of $\sigma_{\xi} \sim \sqrt{3} / \gamma_{n} \sim(\nu / 2)^{1 / 3}$.

We can now interpret these results from a physical point of view. Cavity shortening is necessary to prevent the optical pulse from building up only at the trail-

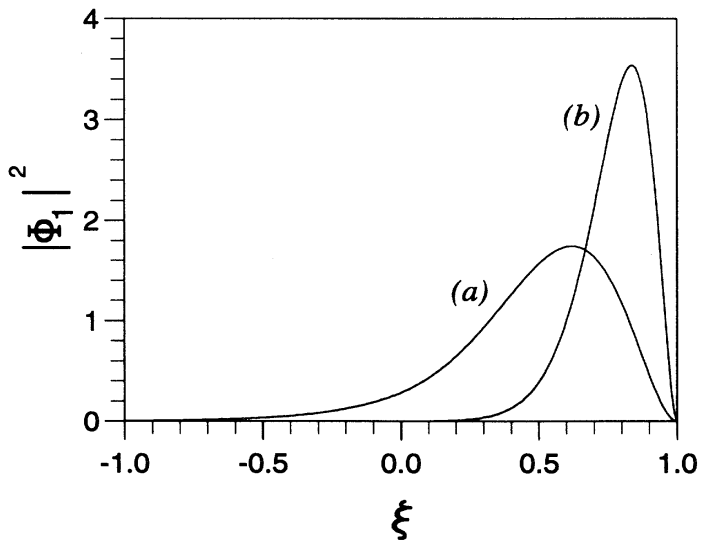

FIG. 2. Supermode $\left|\Phi_{1}\right|^{2}$ vs $\xi$ for (a) $\nu=0.045$ and (b) $\nu=0.005$. 


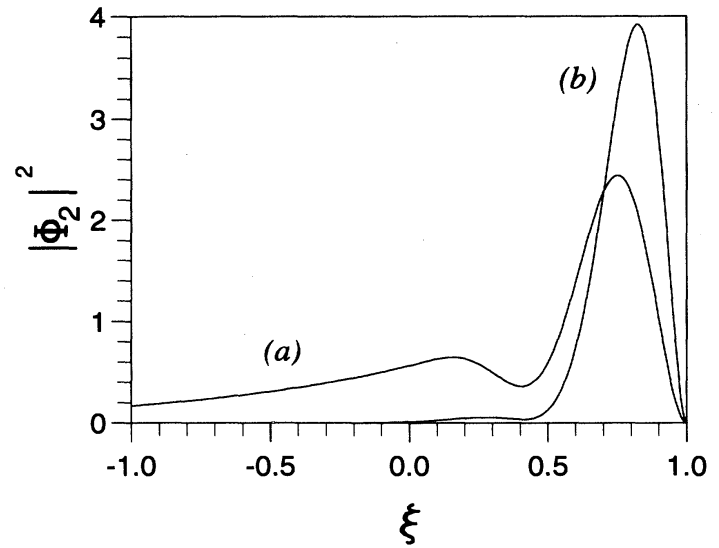

FIG. 3. Supermode $\left|\Phi_{2}\right|^{2}$ vs $\xi$ for (a) $\nu=0.015$ and (b) $\nu=0.005$.

ing edge, with the peak at $\xi=1$ [9]. This effect, called lethargy, is a result of the field building up due to a swept gain [20], with an exponential growth rate proportional to $\tau^{1 / 3}$ instead of $\tau$. When the cavity is shortened with respect to vacuum synchronism, $\nu>0$, the optical pulse is pushed forward and the radiation peaks at $\xi<1$. As the electrons interact with the pulse only for a short time as they approach the end of the undulator the effect of lethargy is relatively small. As a result, a balance between lethargy, which retards the optical pulse, and cavity detuning, which pushes the radiation pulse forward, is achieved and a stationary radiation profile grows exponentially with $\tau$. As the cavity length is further decreased, the radiation pulse widens so as to maintain the balance between cavity shortening and lethargy. Finally, for $\nu>0.13$ the cavity is too short to be counteracted by lethargy, and no stable lasing is possible.

Similar results have been obtained for a parabolic electron bunch shape much longer than the slippage

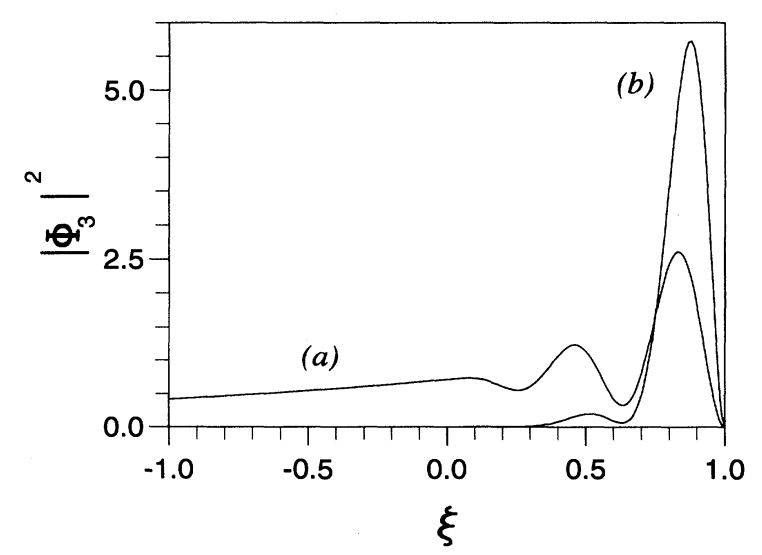

FIG. 4. Supermode $\left|\Phi_{3}\right|^{2}$ vs $\xi$ for (a) $\nu=0.008$ and (b) $\nu=0.002$. length $\left(L_{b} \gg \Delta\right)[14,15]$, showing that stable gain occurs for $2 \delta \mathcal{L} / g_{0} \Delta<0.56$, and a maximum in the gain $\mathcal{G} \simeq 0.27 g_{0}\left(1-0.2 \Delta / L_{b}\right)$ occurs at $2 \delta \mathcal{L} / g_{0} \Delta=0.28$, with a gain curve versus cavity detuning similar to that of Fig. 1. In the long-beam analysis of Ref. [14], the cavity shortening scales as the slippage length $\Delta$ instead of the beam length $L_{b}$, as in our case, and the gain is equal to the $\mathrm{cw}$ gain with a small correction for the effect of the finite bunch length.

The existence of multiple supermodes (with positive linear gain for $\nu<0.02$ ) suggests that nonlinear coupling between different supermodes occurs at saturation, resulting in limit-cycle oscillations and chaotic behavior. In contrast, it is expected that for large cavity shortening $\nu$, the optical field growth will be well described by a single supermode, with saturation at a steady-state value. This situation is in agreement with numerical simulations [6] and the results of several FEL experiments driven by electron beams with a micropulse lengths comparable to or shorter than the slippage length [21-23].

\section{SINGLE SUPERMODE OPERATION}

When the real part of the first eigenvalue is close to the loss, the first supermode is also close to the FEL instability threshold, while all the other supermodes are strongly damped $\left(\left|\operatorname{Re} \mu_{1}-\alpha / 2\right| \ll\left|\operatorname{Re} \mu_{n}-\alpha / 2\right|\right.$, for $\left.n \geq 2\right)$. For this case one can assume that $A(\xi, \tau)=W_{1}(\tau) \Phi_{1}(\xi)$, where $\Phi_{1}$ is the eigenfunction relative to the first eigenvalue $\mu_{1}$ of the linear regime. Substituting this expression in Eqs. (16)-(20) a long but straightforward calculation, up to third order in $W$, gives the following LandauGinzburg equation:

$$
\dot{W}_{1}=\left(\mu_{1}-\alpha / 2\right) W_{1}-\beta_{1} W_{1}\left|W_{1}\right|^{2},
$$

where the overdot stands for a $d / d \tau$ and

$$
\beta_{1}=\int_{0}^{1} d \xi \Phi_{1}^{*}(\xi) \int_{0}^{\xi} d \xi^{\prime}\left(\xi-\xi^{\prime}\right) K\left(\xi^{\prime}\right),
$$

where

$$
\begin{aligned}
K= & 4 \Gamma_{1} \operatorname{Re}\left(\mu_{1}\right) \int_{0}^{\xi} d \xi^{\prime}\left|\Phi_{1}\left(\xi^{\prime}\right)\right|^{2} \\
& -2 \Psi_{1}\left|\Gamma_{1}\right|^{2}-2 \nu \Gamma_{1}\left[\left|\Phi_{1}\right|^{2}-\left|\Phi_{1}(0)\right|^{2}\right]
\end{aligned}
$$

with $\Psi_{n}=\mu_{n} \Phi_{n}-\nu \Phi_{n}^{\prime}$ and $\Gamma_{n}=i \Psi_{n}^{\prime}$ obtained from Eqs. (25)-(30). Setting $W_{1}=I^{1 / 2} \exp \left(i \phi_{1}\right)$, Eq. (42) can be separated into two decoupled equations,

$$
\dot{I}=\left[g-2 \operatorname{Re}\left(\beta_{1}\right) I\right] I
$$

and

$$
\dot{\phi}_{1}=\operatorname{Im} \mu_{1}-\operatorname{Im}\left(\beta_{1}\right) I,
$$

where $g=2 \operatorname{Re} \mu_{1}-\alpha$. At the threshold $g=0$, the system undergoes a Hopf bifurcation, while, for $g>0$, Eq. (45) 
has the following solution:

$$
I(\tau)=\frac{I(0) \exp (g \tau)}{1+\left[I(0) / I_{s}\right][\exp (g \tau)-1]},
$$

where

$$
I_{s}=\frac{g}{2 \operatorname{Re} \beta_{1}}
$$

Using Eqs. (22) and (24), the saturation energy in the single supermode approximation is

$$
\mathcal{E}_{s}=I_{s}\left[1+\frac{\nu\left|\Phi_{1}(0)\right|^{2}}{\alpha}\right],
$$

where the second term is the energy outside and ahead of the slippage region. In Fig. 5 we plot the gain, $\mathcal{G}_{1} / \gamma=2 \operatorname{Re} \mu_{1}$ (a) and the saturated energy for the first supermode $\mathcal{E}_{s}$ (b) as a function of the cavity detuning $\nu$, for three different values of loss $\alpha$. The intensity increases when the cavity loss decreases, showing a sharp peak near $\nu=0$. For a relatively large loss $(\alpha>0.1)$, no pronounced peak in the intensity is observed in the detuning curve, while for a smaller $\alpha$, emission can occur at a smaller cavity detuning, although with a lower gain.
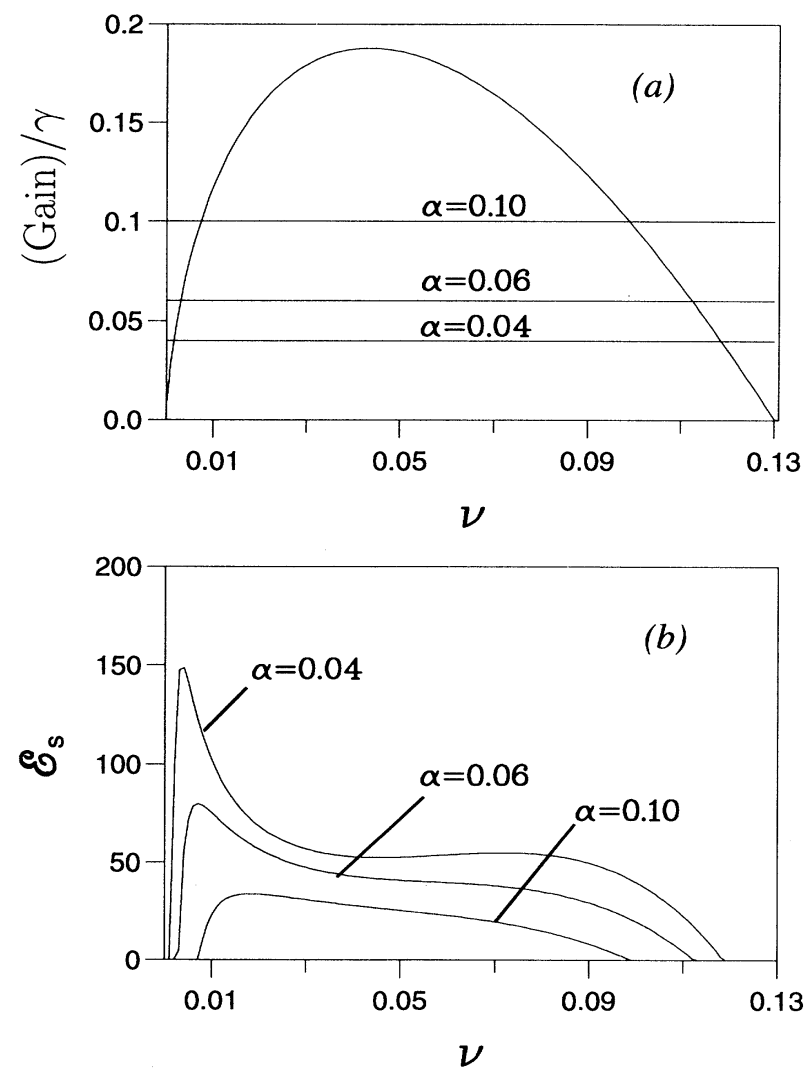

FIG. 5. (a) Linear gain $\mathcal{G}_{1} / \gamma=2 \operatorname{Re} \mu_{1}$ and (b) steady-state energy $\mathcal{E}_{s}$, from Eq. (48), vs cavity detuning $\nu$ for the fundamental supermode and for $\alpha=0.10,0.06$, and 0.04 .
The smaller bump in $\mathcal{E}_{s}$ at larger $\nu$ is due to the factor $1+\nu\left|\Phi_{1}(0)\right|^{2} / \alpha$, which accounts for free propagation of the radiation ahead of the electrons, outside the slippage region.

We show in the following that near vacuum cavity synchronism, at the optimum cavity detuning of Fig. 5(b), the emission is superradiant, with a maximum efficiency scaling as $\sqrt{\mathcal{E}_{e}}$ and a peak power scaling as $\mathcal{E}_{e}^{2}$, where $\mathcal{E}_{e}=P_{e}\left(L_{b} / c\right)$ is the electron micropulse energy. Using the approximate expression for the $n$th supermode in the limit of small $\nu, \Phi_{n} \simeq$ $\Phi_{N} \sin (n \pi \xi) \exp (k \xi), \Psi_{n} \simeq(\nu / 2)^{2 / 3} \exp (i \pi / 6) \Phi_{n}$ and $\Gamma_{n} \simeq-(\nu / 2)^{1 / 3} \exp (-i \pi / 6) \Phi_{n}$, where $\Phi_{N}$ is the normalization constant and $k=(2 / \nu)^{1 / 3} \exp (i \pi / 6)$, a long but straightforward calculation gives $\operatorname{Re} \beta_{1} \simeq(\sqrt{3} / 2) \nu \operatorname{Re} \mu_{1}$. Then, using the approximate expression (36), we obtain, for the first supermode,

$$
I_{s}=\frac{2}{\sqrt{3} \nu}\left(1-\frac{\alpha}{2 \operatorname{Re} \mu_{1}}\right) \simeq \frac{3 \sqrt{3}(\nu / 2)^{2 / 3}-\alpha}{9(\nu / 2)^{5 / 3}}
$$

In Fig. 6 we compare the approximate solution Eq. (50) (dashed line) with Eq. (48) for $\alpha=0.03$. Equation (50) has a maximum $I_{s} \simeq \alpha^{-3 / 2}$ at $\nu \sim 0.363 \alpha^{3 / 2}$. Therefore, using Eq. (23), the maximum efficiency is

$$
\eta=\frac{\alpha I_{s}}{4 \pi N_{w}} \simeq \frac{1}{4 \pi N_{w} \sqrt{\alpha}} \simeq \frac{4 \pi a_{w} F}{1+a_{w}^{2}} \sqrt{\frac{f r_{e}}{\alpha_{0} Z_{R}} \frac{\mathcal{E}_{e}}{m c^{2}}} .
$$

In this expression $r_{e}=e^{2} / 4 \pi \epsilon_{0} m c^{2}$ is the classic electron radius and $Z_{R}=\pi r_{b}^{2} / \lambda$ is the Rayleigh range. Using Eq. (37) for $\left|\Phi_{1}\right|^{2}$ at the optimum cavity detuning $\nu=$ $0.363 \alpha^{3 / 2}$, the peak power is $|A|_{\max }^{2} \sim 0.8 / \alpha^{2}$ and the width is $\sigma_{\xi} \sim 0.56 \sqrt{\alpha}$. As a result, the optical spike is $N_{s}=N_{w} \sigma_{\xi}=0.56 N_{w} \sqrt{\alpha}$ optical periods long and is produced with an efficiency of

$$
\eta \simeq \frac{1}{8 \pi N_{s}}=\rho \sqrt{\frac{L_{b}}{\alpha_{0} L_{c}}} .
$$

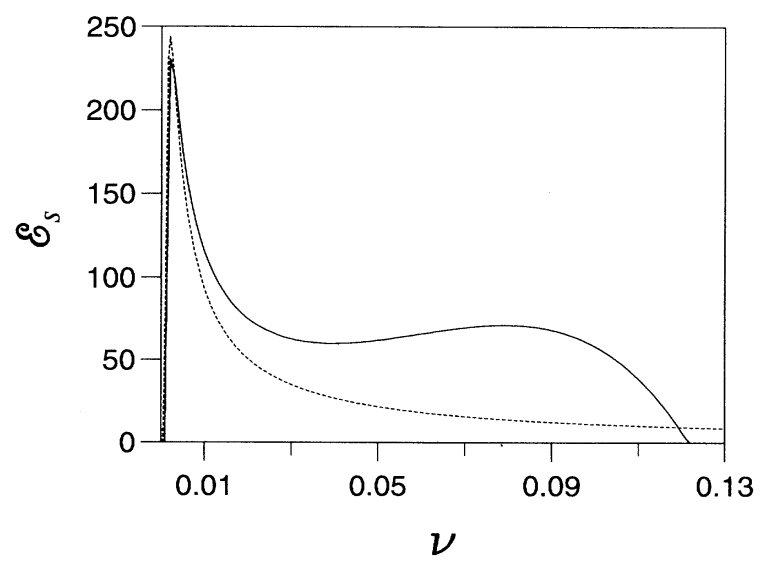

FIG. 6. Comparison between the steady-state energy $\mathcal{E}_{s}$ calculated from Eq. (48) (continuous line) and from the approximate expression, Eq. (50) (dashed line), for $\alpha=0.03$. 
Using the definition of $A$ and $\alpha$, the peak power is

$$
P_{s} \simeq \rho P_{e}\left(\frac{L_{b}}{\alpha_{0} L_{c}}\right)^{2} \simeq \frac{e^{2} c f}{\epsilon_{0} \lambda Z_{R}}\left(\frac{a_{w} F}{1+a_{w}^{2}} \frac{\mathcal{E}_{e}}{m c^{2} \alpha_{0}}\right)^{2}
$$

These expressions are in agreement to within a numerical factor of the order of 1 , with those derived in Ref. [17] with the assumption that the optical power is such that the electrons undergo half a synchrotron oscillation during the interaction time, which is limited by the optical pulse length and not the undulator length. The electrons interact with the optical spike and undergo half an oscillation while traversing $N_{s} \ll N_{w}$ undulator periods so that the maximum efficiency $\eta$ is of the order of $1 / N_{s}$ instead of $1 / N_{w}$ as in a cw FEL $[17,24]$.

Superradiance can be interpreted as the regime in which the radiation extracts energy from the electrons with a maximum efficiency. The same regime has been found in the single-pass high-gain FEL driven by electron bunches with a length shorter than or comparable with the slippage length $[3,18,19]$. A single superradiant spike of peak power $P \simeq 0.2 \rho P_{e}\left(L_{b} / L_{c}\right)^{2}$ is generated by a short electron bunch (weak superradiance), whereas a burst of random spikes is produced by a long electron bunch when the emission starts from shot noise [25]; the same mechanism has been observed in FEL oscillators, where a single spike is generated for electron bunches shorter than the slippage length [21] and for long electron pulses, where a chaotic superposition of spikes has been observed experimentally and numerically in the "postsideband" regime [26]. In the superradiating FEL oscillator, with a cavity length close to that for vacuum synchronism, the efficiency and peak power scale, respectively, as the square root and the square of the quality factor $Q=1 / \alpha_{0}$. For long electron bunches, a spike is produced approximately each slippage length $\Delta$. Reference [26] reports the same calculated efficiency as Eq. (51), within a numerical factor (equal to $4 \sqrt{2}$ ), if $L_{b}$ is substituted for the slippage length $\Delta$. Hence the efficiency for long electron pulses is in general larger than that for short electron pulses and the optical field has chaotic temporal and spectral features. In contrast, short electron pulse FEL's have the advantage of generating an extremely short radiation spike (with a typical length less than $1 \mathrm{ps}$ ) with considerable efficiency and smooth spectra.

In conclusion, for small cavity detuning electrons emit a superradiant spike with a peak power proportional to $\mathcal{E}_{e}^{2}$ and a width inversely proportional to $\sqrt{\mathcal{E}_{e}}$. As a numerical example, for an undulator with $\lambda_{w}=6.5 \mathrm{~cm}$, $a_{w}=1.2$, and $N_{w}=38$, a beam with energy $E=22.5$ $\mathrm{MeV}$, charge $Q=40 \mathrm{pC}$, and $L_{b}=0.5 \Delta, Z_{R}=1.2$ $\mathrm{m}$, filling factor $f=1$, and cavity losses $\alpha_{0}=5 \%$, an optical spike is emitted at $\lambda=40 \mu \mathrm{m}$ with efficiency $\eta \sim 1 \%$, intra-cavity peak power $P_{s}=120 \mathrm{MW}$ and duration $\tau_{s}=0.63 \mathrm{ps}\left(N_{s}=4.75\right)$; the optimum cavity shortening is $\delta \mathcal{L}=3 \mu \mathrm{m}$ and the gain per pass $7 \%$.

\section{MODE-COUPLING AND LIMIT-CYCLE OSCILLATIONS}

Single mode operation leads to a stable configuration without the oscillation of the radiation energy as a function of the scaled pass number $\tau$. However, it has been observed experimentally [27] and numerically [6] that a limit cycle occurs at moderate values of $\nu$ and the macropulse power oscillates periodically over several hundred passes, as does the optical micropulse shape and spectrum. For smaller cavity detuning and loss, period doubling of the oscillations and transition to a chaotic regime occurs as has been discussed in Ref. [7]. In Ref. [7], the transition has been studied numerically by varying three parameters, cavity detuning, beam length, and beam current. In this case, the different regimes are described in terms of the quantity $\mu_{\mathrm{syn}}=\Delta / L_{\mathrm{syn}}=$ $|A|^{1 / 2} / \sqrt{2} \pi$, equal to the number of synchrotron oscillations in a slippage length, where $L_{\mathrm{syn}}(\xi, \tau)$ is the "instantaneous" period of the synchrotron oscillation [see Eq. (12)]. However, this parameter depends on the field strength $|A|$, which must be calculated by numerically integrating the partial differential equations. In contrast, by restricting our model to electron pulses shorter than the slippage length, we have developed a self-consistent analysis of the different regimes of operation of the FEL, which depends only on two (experimentally controllable) parameters, cavity detuning and loss. We find that the wealth of nonlinear FEL physics can be rather accurately described by the reduced set of Eqs. (16)-(20). More importantly, the analysis of the linear supermodes given in Sec. IV provides a convenient framework for understanding such nonlinear phenomena as the limit cycle and chaotic behavior, as well as giving a rather accurate estimate of the frequency of the limit cycle. In this framework, the transition from stable operation to a limit cycle can be interpreted in terms of nonlinear coupling between different supermodes. Nonlinear oscillation of the radiation power results from a competition between different supermodes, whereas the quasi steady-state operation occurs when the first supermode is dominant. When two supermodes dominate, a limit cycle or damped oscillations are observed.

In this section we present numerical results obtained from solving the partial differential system of Eqs. (16)(20). The numerical solution can then be decomposed into the supermodes, $A(\xi, \tau)=\sum_{n} W_{n}(\tau) \Phi_{n}(\xi)$, and the evolution of the coefficients $W_{n}(\tau)$ investigated. We determine the regions in the parameter space, $(\nu, \alpha)$, where stable operation, damped oscillations, a limit cycle or period doubling occurs. The expansion in eigenmodes loses its relevance when the behavior becomes highly nonlinear, because nonlinear coupling between the modes assures a nonvanishing content of every mode in $A(\xi, \tau)$. On the other hand, if all the modes apart from the fundamental are heavily damped, as in the linear regime, and if the saturated amplitude is not too large (i.e., nonlinear effects are small), then the fundamental supermode provides a good approximation for the total field $A$, and the spectrum of the field has a single narrow line at $\omega_{1}=\dot{\phi}_{1}$, 


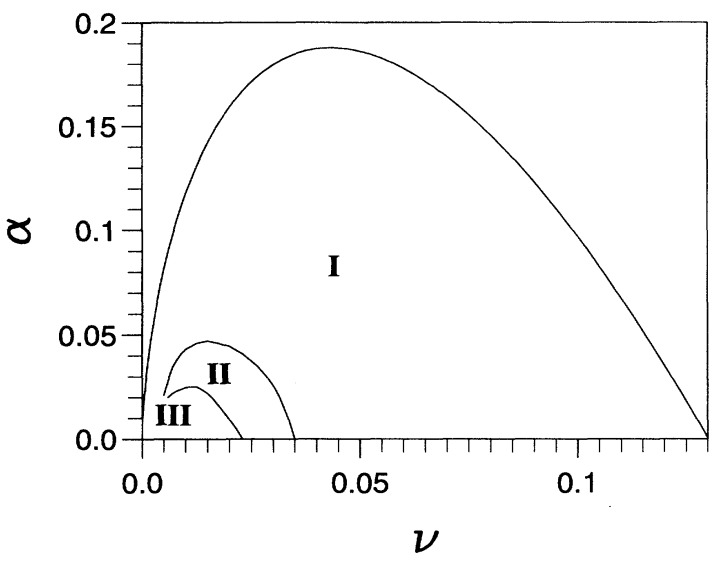

FIG. 7. Phase diagram in the parameter space $(\nu, \alpha)$. Region I: quasistable regime; region II: limit-cycle regime; region III: period-doubling and chaotic regime.

where $\dot{\phi}_{1}$ is given by Eq. (46). This is indeed confirmed by our numerical simulations, which show a single spectral line in the case of a steady-state operation. As the optical power is increased, by decreasing cavity detuning or loss, the second mode is driven nonlinearly, and a second narrow line appears in the spectrum at a frequency $\omega_{2} \approx \operatorname{Im} \mu_{2}$, with a small correction due to a nonlinear phase shift resulting from an interaction with the first mode. The appearance of the second spectral line coincides with the onset of the limit-cycle behavior. Therefore, more generally, the effective number of modes participating in the interaction corresponds to the number of (independent) spectral lines.

Figure 7 shows a phase diagram in $\alpha$ and $\nu$ of the different regimes: no emission occurs above the threshold, for $\alpha>\mathcal{G}_{1} / \gamma$, whereas quasi-steady-state operation or damped oscillations occur in region I of Fig. 7, with a very small amplitude high-frequency oscillation; limit cycles, at a lower frequency, are observed in region II, whereas period-doubling behavior occurs in the transition region between II and III. In region III, a transition to chaotic behavior occurs via a period-doubling cascade; a detailed study of this latter regime is beyond the scope of this paper. In the following we discuss the different regimes separately.

\section{A. Quasistable operation}

In the quasistable regime the radiation energy reaches a quasistable equilibrium, with a small oscillation at the single-mode frequency $\dot{\phi}_{1}$ of Eq. (46). In Fig. 8(a) (continuous line) we present the temporal evolution of the rms field within the slippage length

$$
\mathcal{A}(\tau)=\left[\int_{0}^{1} d \xi|A(\xi, \tau)|^{2}\right]^{1 / 2}
$$

calculated from the numerical simulation for $\alpha=0.14$ and $\nu=0.05$. We have chosen initial and boundary conditions as explained in Sec. IV, with $A_{0}=0.01$. The other curves of Fig. 8(a) represent the temporal evolution of the amplitudes $\left|W_{1}\right|$ (dashed line) $\left|W_{2}\right|$ (dotted line), and $\left|W_{3}\right|$ (dashed-dotted line) of the first three supermodes, calculated by projecting the numerical solution $A(\xi, \tau)$ on the eigenfunctions, $\Phi_{1}, \Phi_{2}$, and $\Phi_{3}$. Due to the nonorthogonality of these functions, the decomposition depends, in general, on the number of eigenfunctions taken into account. In the linear regime, $\left|W_{1}\right|$ grows exponentially with a rate $\operatorname{Re} \mu_{1}$, whereas the other two modes are damped. In the nonlinear regime the first supermode drives the other two modes at a rate three times larger, due to a pump term proportional to $\left|W_{1}\right|^{2} W_{1}$. The three amplitudes reach saturation at different values, with the first supermode dominating, and the other two saturating well below the average amplitude. Although not visible in the figure, the first supermode amplitude has a small oscillation, of order $10^{-3}$, at the frequency $\dot{\phi}_{1}$ (46). This oscillation results from the beating between the steady-state solution (driven by the incoming spontaneous emission at $\xi=1$ ) and the first mode. In real experiment this coherent oscillation is difficult to observe
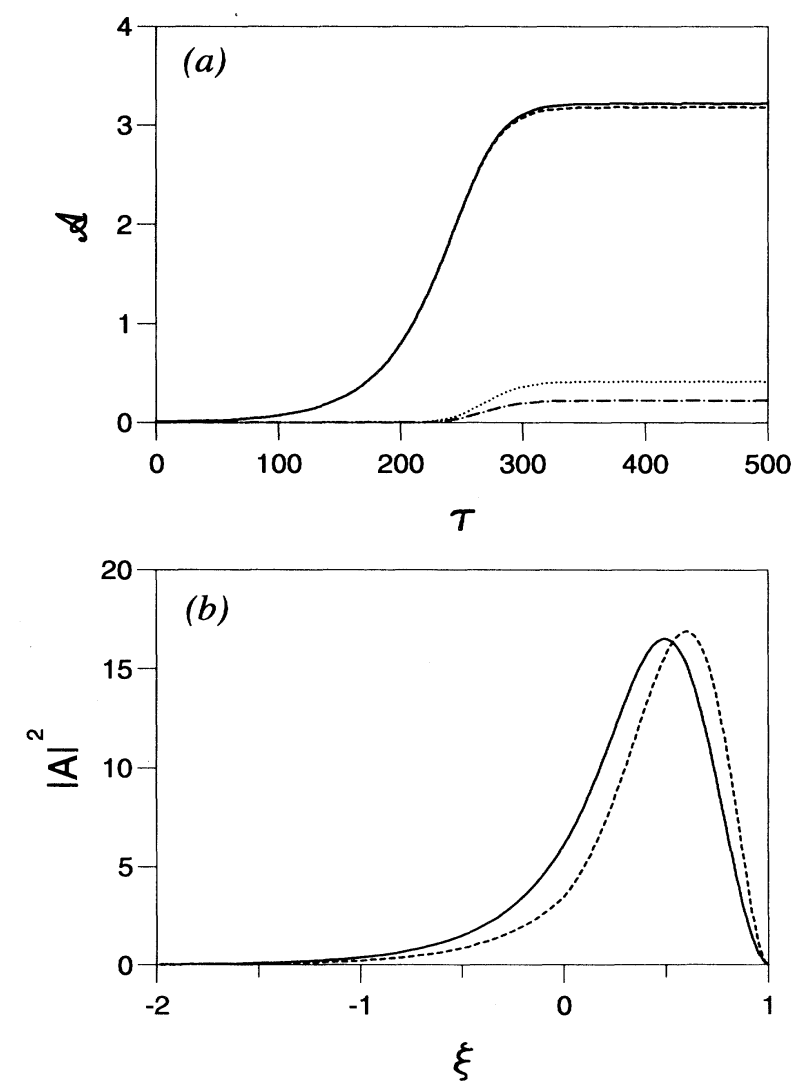

FIG. 8. Steady-state regime. (a) Average field $\mathcal{A}$ vs $\tau$ for $\alpha=0.14$ and $\nu=0.05$; dashed line: $\left|W_{1}\right|$; dotted line: $\left|W_{2}\right|$; dashed-dotted line: $\left|W_{3}\right|$. (b): steady-state power profile $|A|^{2}$ (continuous line) and single mode approximation $|A|^{2}=\left|W_{1} \Phi_{1}\right|^{2}$ (dashed line) vs $\xi$. 


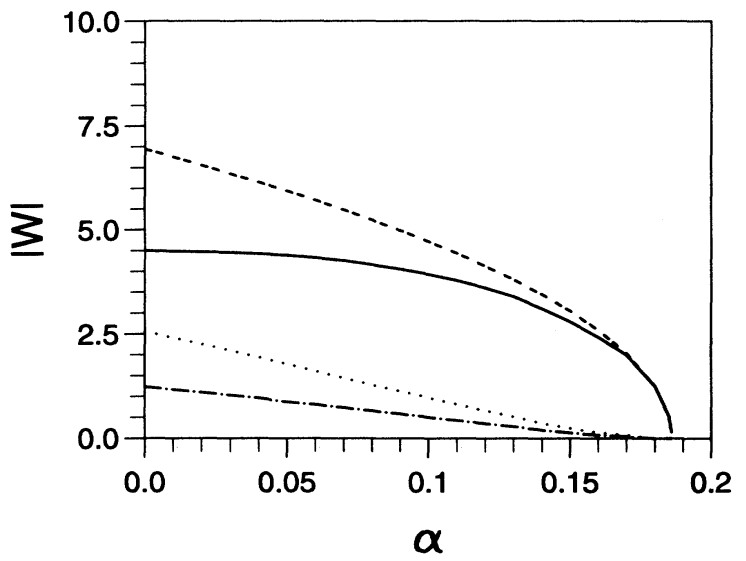

FIG. 9. Steady-state regime. Supermode amplitudes $\left|W_{n}\right|$ at saturation as a function of $\alpha$ for $\nu=0.05$; continuous line: $n=1$; dotted line: $n=2$; dashed-dotted line: $n=3$; dashed line: analytical solution, Eq. (48), in the single-mode approximation.

because its magnitude relative to the saturated power is typically very small $[21,28]$.

The optical intensity $|A|^{2}$ at saturation is shown in Fig. 8(b) (continuous line) as a function of $\xi$, together with the single-mode approximate solution $|A|^{2}=\left|W_{1} \Phi_{1}\right|^{2}$ (dashed line), where $W_{1}$ is calculated numerically. In this case the saturated optical field is almost proportional to the first supermode, and the higher supermodes can be neglected. Figure 9 shows the steady-state value of the three amplitudes $\left|W_{1}\right|$ (continuous line), $\left|W_{2}\right|$ (dotted line), and $\left|W_{3}\right|$ (dashed-dotted line), as a function of $\alpha$ for $\nu=0.05$, as they result from the expansion of the numerical solution on the first three supermodes. The dashed line represents the single-supermode solution, Eq. (48), $\left|W_{1}\right|=\sqrt{I_{s}}$. This expression is in agreement with the numerical value of $\left|W_{1}\right|$ for values of $\alpha$ near the threshold $\alpha=0.186$. By decreasing $\alpha$, the amplitudes of the other supermodes increase, reducing the value of $\left|W_{1}\right|$ from that of the single-mode solution. This further illustrates our previous comment that the structure of the nonlinear saturated field can somewhat deviate from the lowest supermode despite the fact that a single frequency is present in the system.

\section{B. Damped oscillation and limit cycle}

When two supermodes grow to a comparable amplitude, a damped or limit-cycle oscillation can occur. We have found numerically that limit-cycle oscillations occur in region II of Fig. 7 bounded by $\nu<0.035$ at $\alpha=0$ and $\alpha<0.047$ at $\nu=0.015$. Crossing the boundary I-II, the damped oscillations become stable and a limit cycle is established. Figure 10 shows the $\operatorname{rms}$ field $\mathcal{A}$ averaged over one limit-cycle period (dashed line) and the maximum and minimum of the oscillation (continuous lines) for $\alpha=0.03$ as a function of $\nu$ (a) and for $\nu=0.025$ as a function of $\alpha(b)$, crossing the boundary I-II. In Fig. 10, the steady-state profile is reached asymptotically for $\nu>0.035$ (a) and $\alpha>0.4$ (b), whereas a bifurcation to a limit cycle occurs for smaller values of $\nu$ and $\alpha$. For these values of $\alpha$ and $\nu$, no period doubling is observed.

As an example, we will analyze in detail how crossing the I-II boundary along the constant cavity detuning curve $\nu=0.03$ for two different values of $\alpha$ on either sides of the I-II boundary takes the oscillator from the regime of damped oscillation to the regime of the limit cycle.

Figure 11(a) shows the rms field $\mathcal{A}$, Eq. (54) (continuous line) and the components of the first three supermodes, as a function of $\tau$ for $\nu=0.03$ and $\alpha=0.04$. The radiation exhibits a damped oscillation, with the amplitude of the third supermode still rather small compared with the average saturation value. Figure 11(b) shows the optical micropulse power profile for the steady state (continuous line) and that for the two-mode approximation, $|A|^{2}=\left|W_{1} \Phi_{1}+W_{2} \Phi_{2}\right|^{2}$ (dashed line). The spectrum of the complex amplitude $A(\xi=0.5, \tau)$ at the midpoint $\xi=0.5$, shown in Fig. $11(\mathrm{c})$, consists of a single line at $\omega_{1}=0.157$, in good agreement with the value $\phi_{1}=0.162$ calculated from Eq. (46). Hence, the sys-
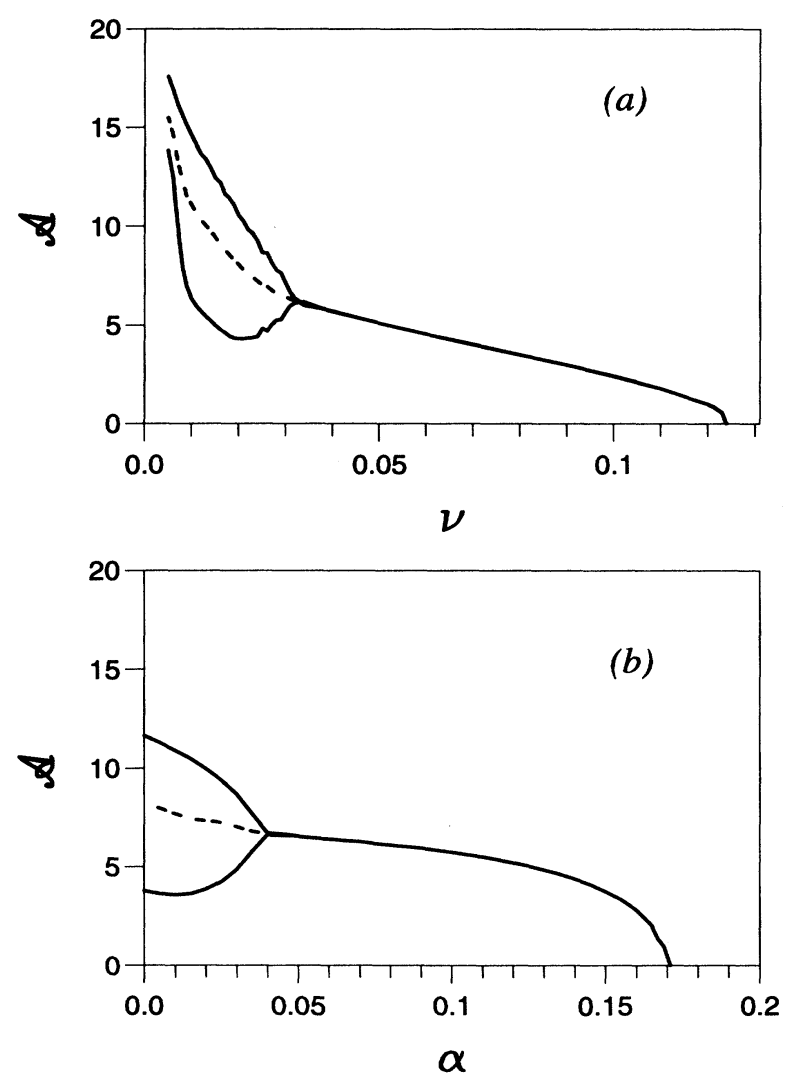

FIG. 10. Limit-cycle oscillation. rms field $\mathcal{A}$, averaged over a limit-cycle period (dashed line) and maximum and minimum amplitudes (continuous lines), (a) vs $\nu$ for $\alpha=0.03$ and (b) vs $\alpha$ for $\nu=0.025$. 

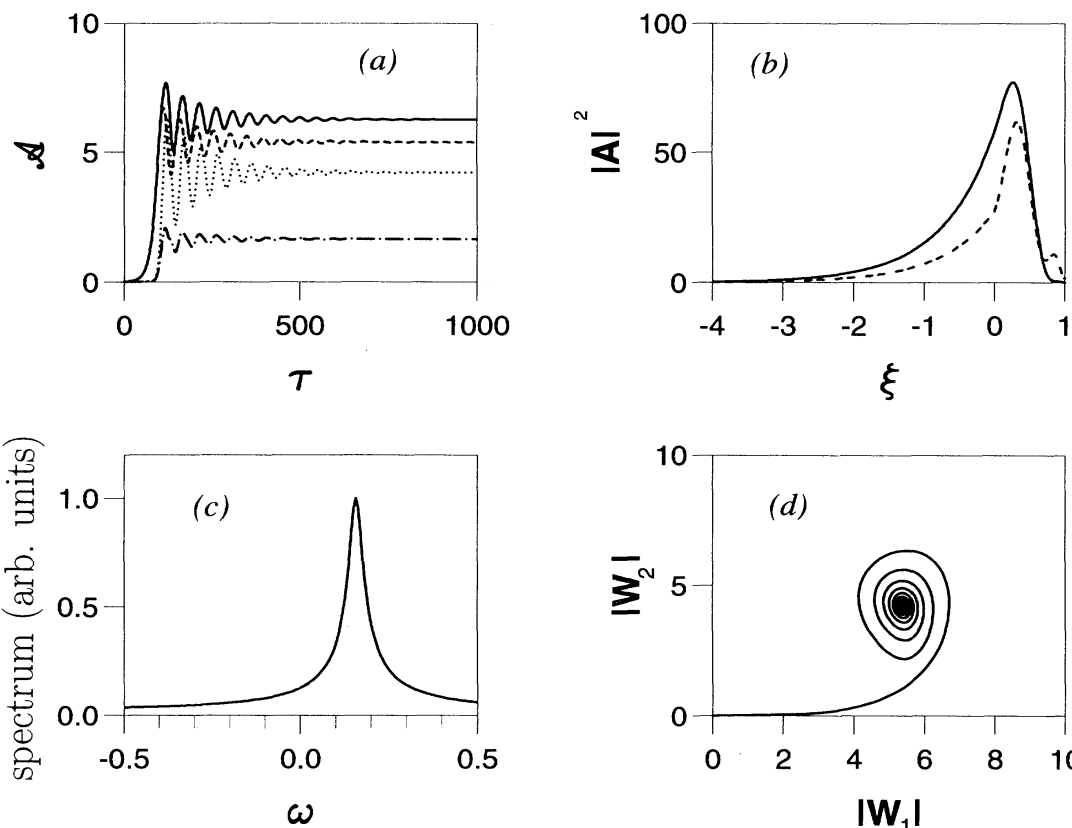

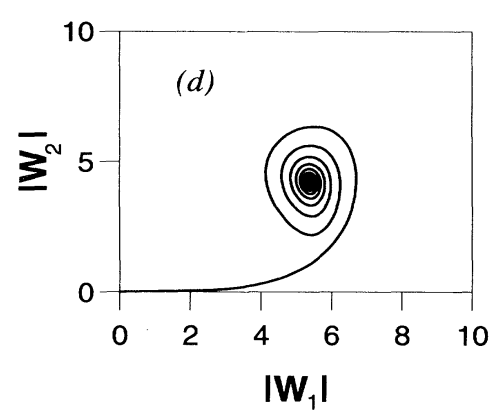

FIG. 11. Damped oscillation regime. (a) Average field $\mathcal{A}$ vs $\tau$ for $\nu=0.03$ and $\alpha=0.04$; dashed line: $\left|W_{1}\right|$; dotted line: $\left|W_{2}\right|$; dashed-dotted line: $\left|W_{3}\right|$; (b) steady-state power profile $|A|^{2}$ (continuous line) and two mode decomposition $|A|^{2}=\left|W_{1} \Phi_{1}+W_{2} \Phi_{2}\right|^{2}$ (dashed line) vs $\xi$ (c) spectrum of the complex amplitude $A$ at the midpoint $\xi=0.5$; (d) $\left|W_{1}\right|-\left|W_{2}\right|$ plane showing the stable point operation (in arbritrary units). tem relaxes to a single-frequency solution. This is also observed in Fig. 11(d) where a stable point is shown in the $\left|W_{1}\right|-\left|W_{2}\right|$ plane. Note that despite the fact that only a principal frequency appears in the spectrum of the steady-state radiation in Fig. 11(c), the longitudinal profile in $\xi$ shown in Fig. 11(b) has a small component of the second supermode.

Figures 12(a)-12(d) show the same case as for Fig. 11, but with a smaller cavity loss, $\alpha=0.02$. A very different picture emerges here: the damped oscillation becomes a limit cycle, still described to a good approximation by two supermodes. The spectrum of the radiation amplitude in Fig. 12(c) shows two main lines at $\omega_{1}=0.157$ and $\omega_{2}=0.283$, associated with the first and the second supermodes, respectively; in fact, Eq. (46) gives $\dot{\phi}_{1}=0.168$, whereas the linear frequency of the second supermode is $\dot{\phi}_{2}=\operatorname{Im} \mu_{2}=0.3$. The difference of $7 \%$ between the analytical and the observed values results from the nonlinear coupling between the two supermodes. The limitcycle frequency of the rms field $\mathcal{A}$ shown in Fig. 12(a)
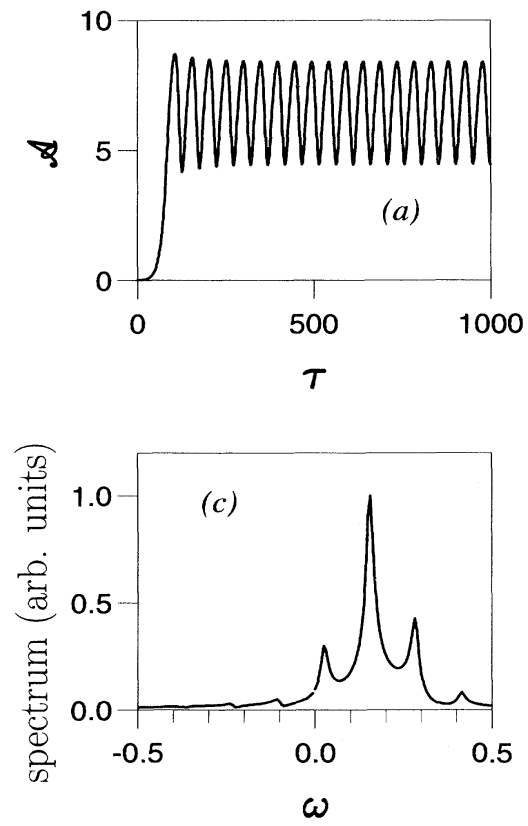
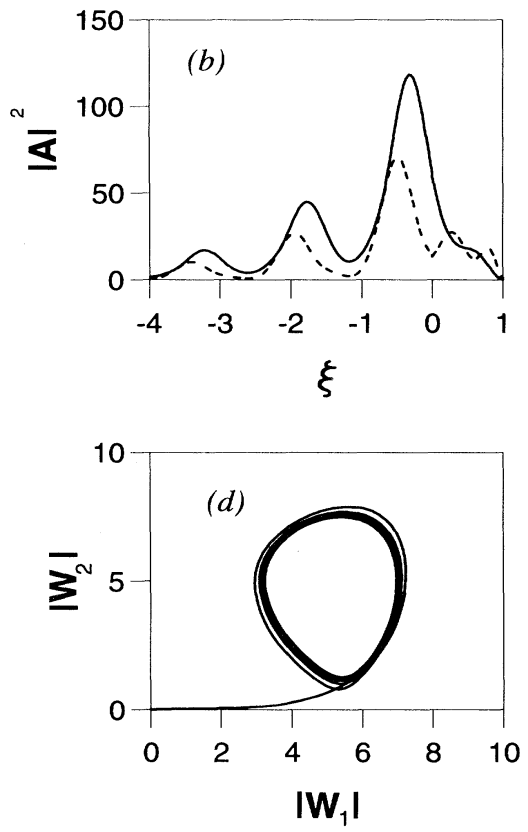

FIG. 12. Limit cycle. Same as in Fig. 11 ut for $\alpha=0.02$ and optical profile deterined at $\tau=1000$. 
is equal to the difference between the frequencies of the two supermodes,

$$
\omega_{\mathrm{LC}}=\omega_{2}-\omega_{1} .
$$

The other main spectral lines appearing in Fig. 12(c) are $\omega=\omega_{1}-\omega_{\mathrm{LC}}=0.03$ and $\omega=\omega_{2}+\omega_{\mathrm{LC}}=0.41$ and all the spectral lines are spaced by $\omega_{\mathrm{LC}}=0.13$.

We note from this example that many of the characteristics of a manifestly nonlinear phenomenon such as limit cycling can be traced to the spectrum of linear supermodes [29]. The frequency of the limit cycle $\omega_{\mathrm{LC}}$ is given fairly accurately as the difference between the frequencies of the lowest supermodes that scale universally with cw gain parameter $g_{0}$, beam length $L_{b}$ (provided $L_{b} \ll \Delta$ ), and cavity detuning $\delta \mathcal{L}$. In Ref. [7] the numerically obtained value of the limit-cycle frequency, $\omega_{\mathrm{LC}}$, is related to the numerically calculated value of the synchrotron frequency. In our analysis, however, there is no need to calculate the amplitude of the radiation numerically in order to predict the frequency of the limit-cycle oscillation for a short electron bunch. Moreover, we find
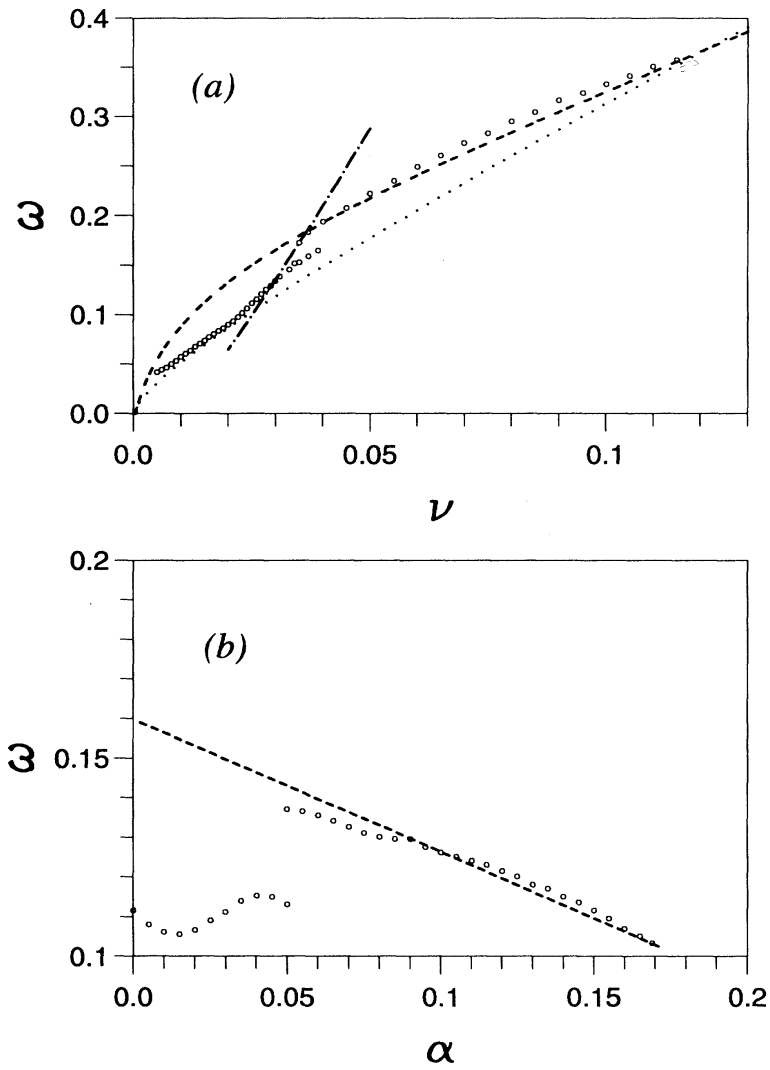

FIG. 13. Oscillation frequency $\omega$ for the same parameters as for Fig. 10. (a) $\omega$ vs $\nu$ (circles), nonlinear single-mode frequency shift $\dot{\phi}_{1}$ (46) (dashed line), $\dot{\phi}_{2}-\dot{\phi}_{1}$ (dashed-dotted line), where $\dot{\phi}_{2}=\operatorname{Im} \mu_{2}$; the linear frequency shift (40) (dotted line) is also shown for reference. (b) $\omega$ vs $\alpha$ (circles) and nonlinear single-mode frequency shift $\dot{\phi}_{1}$ (46) (dashed line). that in the case of a short electron bunch the concept of synchrotron frequency is not very useful. The supermode approach is more convenient because of the large separation between supermodes, which allows one to reduce the effective number of degrees of freedom. For a long pulse a large number of supermodes can be simultaneously unstable [15], and the concept of synchrotron oscillation again becomes relevant.

In Fig. 13 the calculated oscillation frequency $\omega$ (circles) vs $\nu$ (a), and vs $\alpha$ (b), is shown for the same cases as Fig. 10. In Fig. 13(a), for $\nu<0.035$, the frequencies (circles) corresponding to the asymptotic stable operation are in good agreement with the single-supermode values given by Eq. (46) (dashed line). Near the value of $\nu$ for which the two supermode frequencies become locked, i.e., $\omega_{2}=2 \omega_{1}$, the frequency deviates from the single-mode value and follows the curve $\dot{\phi}_{2}-\dot{\phi}_{1}$ (dashed-dotted line), where $\dot{\phi}_{2}\left(=\operatorname{Im} \mu_{2}\right)$ is the linear frequency and $\dot{\phi}_{1}$ is given by Eq. (46). When $\nu$ is decreased further, the nonlinear coupling between the two supermodes becomes more important and the frequencies of the two supermodes deviate from their respective single-supermode values. In
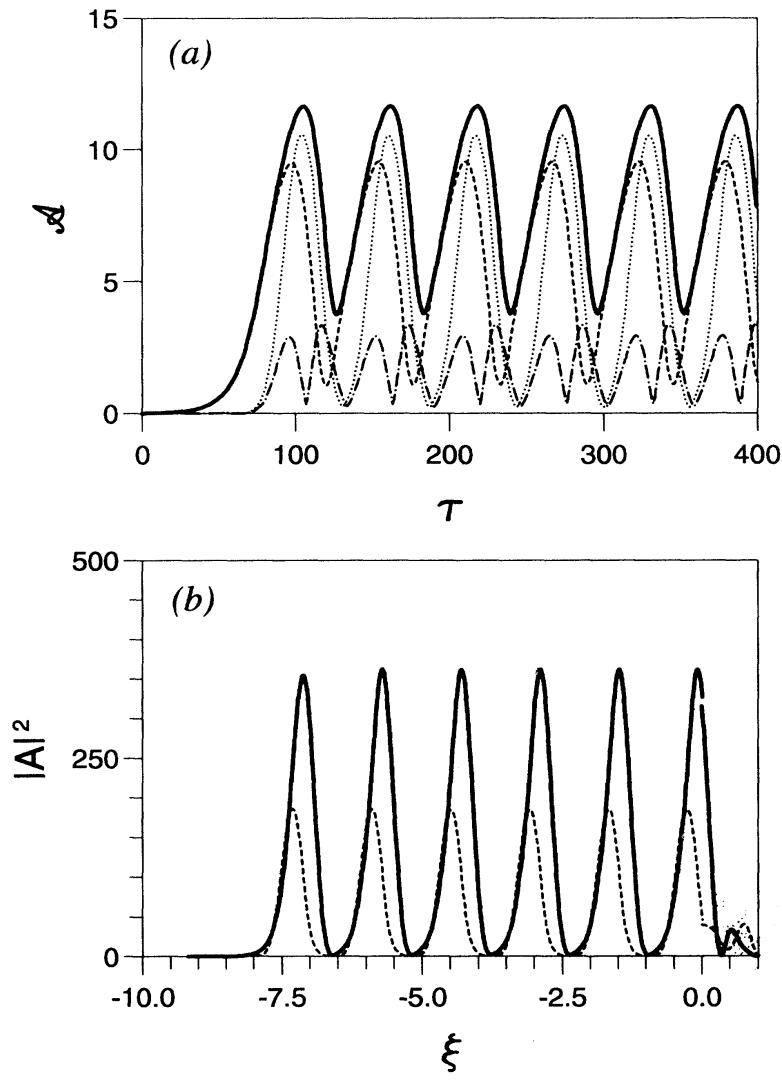

FIG. 14. Limit-cycle regime. (a) Average field $\mathcal{A}$ vs $\tau$ for $\alpha=0$ and $\nu=0.025$; dashed line: $\left|W_{1}\right|$; dotted line: $\left|W_{2}\right|$; dashed-dotted line: $\left|W_{3}\right|$. (b) Steady-state power profile $|A|^{2}$ (continuous line) and two mode decomposition $|A|^{2}=\left|W_{1} \Phi_{1}+W_{2} \Phi_{2}\right|^{2}$ (dashed line) vs $\xi$ at $\tau=400$. 
the transition region of Fig. 13(a), for $0.033<\nu<0.039$, we show the asymptotic frequency (upper branch) and the damped frequency (lower branch). The damped oscillation becomes a limit cycle and the branches merge to the same value at $\nu=0.033$. In Fig. 13(a), the linear value $\omega_{1 L}=\operatorname{Im} \mu_{1}$, for the first supermode (dotted line), is drawn for reference. We find that the numerically calculated values of the limit-cycle frequency $\omega_{\mathrm{LC}}$ are always slightly larger than the linear frequency $\omega_{1 L}$. From the numerical results, we can estimate the number of round-trip cavity passes for one-limit-cycle period as

$$
\begin{aligned}
n_{L C} & \sim \frac{2 \pi}{\gamma \omega_{L C}} \simeq \frac{4 \pi}{3 \gamma(\nu / 2)^{2 / 3}\left[1+\pi^{2}(\nu / 2)^{2 / 3}\right]} \\
& \simeq \frac{4 \pi L_{c}}{3 L_{b}^{1 / 3} \delta \mathcal{L}^{2 / 3}},
\end{aligned}
$$

where in the final expression the approximate Equation (40) for $\omega_{1 L}$ has been used. The limit-cycle period is inversely proportional to the number of cooperation lengths $L_{c}$ contained in the effective interaction length $L_{b}^{1 / 3} \delta \mathcal{L}^{2 / 3}$ and inversely proportional to the gain $\mathcal{G}$ [see Eq. (41)].

In Fig. 13(b) the oscillation frequency is shown versus $\alpha$, for $\nu=0.025$. In the single-mode operation, for $\alpha>$ $0.09, \omega$ decreases linearly with $\alpha$ in agreement with Eqs. (46) and (48), whereas the damped oscillation becomes a limit cycle for $\alpha<0.05$, with the limit-cycle frequency almost independent of $\alpha$.

Finally, to show that, for very small loss, $\alpha \ll 1$, a very long mode-locked optical pulse train can arise (which can eventually be cavity dumped), Fig. 14(a) shows the rms field $\mathcal{A}$ (continuous line) and the components of the first three supermodes as a function of $\tau$ for $\nu=0.025$ and $\alpha=0$. Figure 14(b) shows the optical power profile (continuous line) and the two-mode approximation, $|A|^{2}=\left|W_{1} \Phi_{1}+W_{2} \Phi_{2}\right|^{2}$ (dashed line). In this case a train of undamped pulses propagates ahead of the electrons (for $-\nu \tau<\xi<0$ ) with a spatial period of $2 \pi \nu / \omega_{\mathrm{LC}}$.

\section{Period-doubling regime}

In the period-doubling regime, the oscillation frequency is sequentially doubled, until the oscillation becomes irregular and the chaotic regime ensues. The period-doubling regime occurs in region III of the phase diagram shown in Fig. 7, for $\nu<0.023$ at $\alpha=0$ and for $\alpha<0.025$ at $\nu=0.012$. As an example of the sequential bifurcations in the oscillation amplitude, Fig. 15 shows the local maxima and minima of the rms field $\mathcal{A}$ as a function of $\alpha$ for $\nu=0.01$; the dashed line shows the rms field averaged over one oscillation period. An almost stable oscillation occurs for $0.05<\alpha<0.1176$, followed by a limit cycle for $0.026<\alpha<0.05$, with a frequency $\omega_{\mathrm{LC}}=0.056$. For smaller values of $\alpha$, the oscillation period is repeatedly doubled, until the oscillation becomes chaotic for $\alpha<0.021$. Figure 15 shows only two sequences of period doubling. From this result we conclude that chaos can be observed for very small values of cavity detuning and loss, where several supermodes are excited in the linear stage of the evolution,

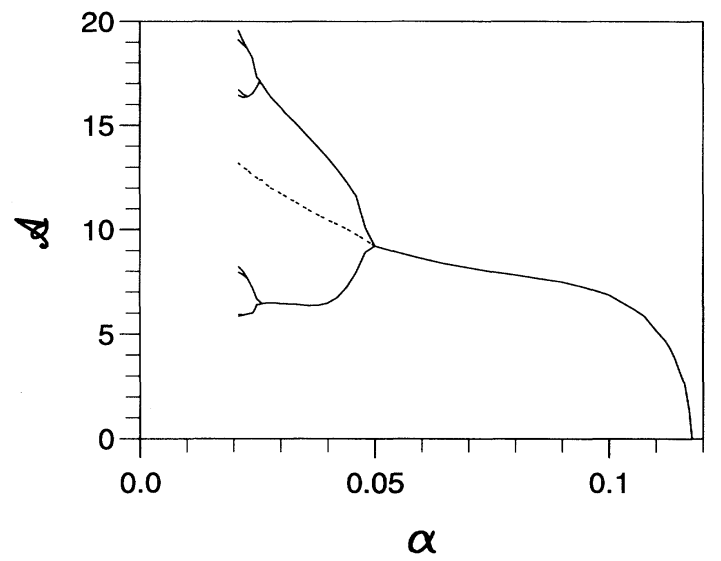

FIG. 15. Bifurcation diagram for $\nu=0.01$. Continuous line: maxima and minima of the $\operatorname{rms}$ field $\mathcal{A}$ vs $\alpha$; dashed line: $\mathcal{A}$ averaged over an oscillation period.

i.e., in the lower left corner of Fig. 7. In this regime the total field is described by more than two supermodes.

\section{COMPARISON WITH FELIX EXPERIMENT AND DISCUSSION}

The first experimental observations of limit-cycle oscillations were made using the free-electron laser FELIX (Free-Electron Laser for Infrared eXperiments) [27] operating in the infrared and far-infrared spectral domains (5-110 $\mu \mathrm{m})$. The observations of limit-cycle oscillations were made possible by the fact that FELIX can operate in the large slippage regime where the slippage length $\Delta$ is greater than the electron bunch length.

In this section we present an analysis of measurements, made on FELIX, of the dependence of the small signal gain, efficiency, and limit-cycle behavior on the cavity detuning. In particular, the previous analysis in terms of supermodes allows one to identify the boundaries between the regions of steady-state operation and limitcycle behavior on FELIX and the conditions under which superradiance can be observed.

FELIX operates as a user facility [21] and consists of two oscillators driven by a $3-\mathrm{GHz} \mathrm{RF}$ linac that produces $10-\mu$ s-long trains of electron bunches, each with a duration of $3 \mathrm{ps,} \mathrm{a} \mathrm{charge} \mathrm{of} 200 \mathrm{pC}$, and an energy in the range of 14-45 MeV. The facility serves the needs of a large and varied scientific community utilizing the tunability, the high peak power and the (subpicosecond) temporal properties of the FEL. Each oscillator consists of a near-concentric optical cavity and a 38-period planar undulator with a periodicity of $65 \mathrm{~mm}$. The undulators have adjustable parameters, $a_{w}<1.9$, allowing the wavelength to be varied over a factor of two for each linac energy. We examine here the behavior of the longerwavelength FEL oscillator, FEL1, that operates in the range $20-110 \mu \mathrm{m}$ and is driven by a single linac section with an energy range of $15-25 \mathrm{MeV}$. The optical cavity 
is $6 \mathrm{~m}$ long and has a Rayleigh range of $Z_{R}=1.2 \mathrm{~m}$, approximately half of the undulator length. The optical radiation is coupled out of the cavity through a small hole, $2 \mathrm{~mm}$ in diameter, situated at the center of one of the gold coated optical cavity mirrors. The cavity length is servo controlled using an interferometer that stabilizes the length to within a fraction of a $\mu \mathrm{m}$ and allows the cavity detuning $\delta \mathcal{L}$ to be systematically scanned. The electron bunch repetition rate is $1 \mathrm{GHz}$, giving 40 concurrently circulating optical pulses in the cavity at any time. FEL1 has a maximum measured small signal gain of $50 \%$ at the peak of the detuning curve and losses ranging from $3 \%$ to $15 \%$ [21]. The small signal gain and loss have been measured using a fast pyroelectric detector $(<200$ ns response time).

In this section we examine the gain, efficiency, and limit-cycle behavior measured at $25 \mu \mathrm{m}\left(a_{w} \approx 0.7\right)$ and at $40 \mu \mathrm{m}\left(a_{w} \approx 1.2\right)$ for an electron energy of $\mathcal{E}_{e} \approx 22$ $\mathrm{MeV}$ and at $60 \mu \mathrm{m}\left(a_{w} \approx 1.0\right)$ for $\mathcal{E}_{e} \approx 16 \mathrm{MeV}$. To determine the net gain $\mathcal{G}-\alpha_{0}$ (well below saturation) at each wavelength as a function of $\delta \mathcal{L}$, the rising and the falling edge of the optical macropulses has been measured and then, using a least-squares fit of the logarithm of the shape, the gain $\mathcal{G}$ and the cavity loss $\alpha_{0}$ per pass are determined.

The gain parameter $\gamma=\left(L_{b} / \Delta\right) g_{0}$ is determined from the peak of the measured gain detuning curve and from the relation $\gamma=5.33 \mathcal{G}_{\text {opt }}$ derived from Eq. (1). A typical gain detuning curve is shown in Fig. 16. In FELIX, the electron bunch shape is approximately triangular with $L_{b} \approx 0.38 \mathrm{~mm}$ [28]. Table I shows the experimentally determined values of $\mathcal{G}_{\text {opt }}, \alpha_{0}, \delta \mathcal{L}_{\text {opt }}$ for each wavelength, together with the corresponding scaled loss $\alpha$ and gain parameter $\gamma$. The uncertainty in the measurements of the losses is about $2 \%$. In Table I we also show the gain parameter as calculated from the definition in Sec. II, with $r_{b}=\sqrt{\lambda Z_{R} / \pi}$ and a phenomenological filling factor, $f=0.356$, the same for each wavelength. Because our simplified model has zero energy spread and emittance, we have introduced a filling factor less than 1, which accounts for the decrease of the small signal gain.

In Fig. 16, the measured gain, divided by $\gamma$, is shown as a function of $\nu=\left(2 / \gamma N_{w}\right)(\delta \mathcal{L} / \lambda)$, together with the gain corresponding to the first supermode (continuous line) and the approximate expression for small $\nu$, Eq. (39), (dashed line). The experimental points fit the first supermode gain well and show the correct scaling with $\gamma$. The optimum detuning length is found to be at $\delta \mathcal{L}_{\text {opt }} \approx$ $0.855 \gamma \lambda$, whereas, for $\delta \mathcal{L}<\delta \mathcal{L}_{\text {opt }}$, the small signal gain per pass depends on $\delta \mathcal{L}$ as $\mathcal{G} \sim 0.0222 \gamma^{1 / 3}(\delta \mathcal{L} / \lambda)^{2 / 3}$,

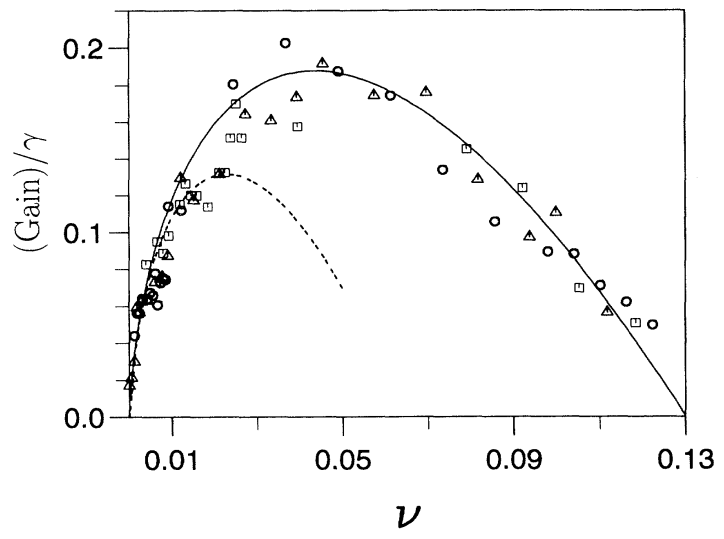

FIG. 16. Comparision with the FELIX experiment. Continuous line: linear gain for the first supermode, $\mathcal{G} / \gamma=2 \operatorname{Re} \mu_{1}$ vs $\nu=\left(2 / \gamma N_{w}\right)(\delta \mathcal{L} / \lambda)$; dashed line: approximated expression, Eq. (39); boxes: $\lambda=25 \mu \mathrm{m}$; circles: $\lambda=40 \mu \mathrm{m}$; triangles: $\lambda=60 \mu \mathrm{m}$.

slightly different from the previously experimentally determined dependence as $(\delta \mathcal{L} / \lambda)^{1 / 2}$ for FELIX [30].

The corresponding efficiency detuning curve for $25 \mu \mathrm{m}$, measured at the end of the macropulse, is shown in Fig. 17 (circles) as a function of $\nu$ over a range of $\delta \mathcal{L}$ between 0 and $4 \lambda$, and compared with the calculated efficiency, obtained by the integration of Eqs. (16)-(20), with $\alpha=\alpha_{0} / \gamma=0.02$ (continuous line). Efficiency values determined from the simulation are shown in Fig. 17 together with the corresponding measured values. The maximum calculated efficiency, $\eta=1.6 \%$ at $\nu=0.004$ $(\delta \mathcal{L}=0.122 \lambda)$, compares well with a maximum measured efficiency of $\eta=1.6 \%$ determined from the electron energy spectra after interaction [31,32]. However, the theoretical curve has a maximum of $3.6 \%$ at $\nu=0.001$ $(\delta \mathcal{L}=0.03 \lambda)$, which is not attainable in the experiment because of the limited electron macropulse length. The uncertainty in the measured vacuum desynchronism length, $\delta \mathcal{L}=0$, as determined from the small signal detuning curve, is $\delta \mathcal{L}= \pm 0.1 \lambda$, corresponding to an uncertainty in $\nu$ of \pm 0.003 , of the same order as that of the optimum $\nu$. In spite of the fact that the optimum cavity shortening can be hidden by the uncertainty in the measurement of $\delta \mathcal{L}$, the efficiency detuning curve fit quite well with the corresponding calculated curve, showing that superradiance should be observable at the smallest $\delta \mathcal{L}$ values.

TABLE I. Parameters of the FELIX experiment.

\begin{tabular}{llrrrrr}
\hline \hline$\lambda(\mu \mathrm{m})$ & $\mathcal{G}_{\text {opt }}(\%)$ & $\delta \mathcal{L}_{\text {opt }}(\mu \mathrm{m})$ & $\alpha_{0}(\%)$ & $\alpha(\%)$ & $\gamma^{\mathrm{a}}$ & $\gamma^{\mathrm{b}}$ \\
\hline 25 & 30.0 & 34.2 & 3.2 & 2.00 & 1.60 & 1.93 \\
40 & 40.3 & 73.5 & 2.9 & 1.35 & 2.15 & 1.80 \\
60 & 27.2 & 74.4 & 2.3 & 1.59 & 1.45 & 1.48 \\
\hline \hline
\end{tabular}

${ }^{a}$ Calculated from the measured peak gain.

${ }^{\mathrm{b}}$ Calculated from the definition, with $f=0.356$. 


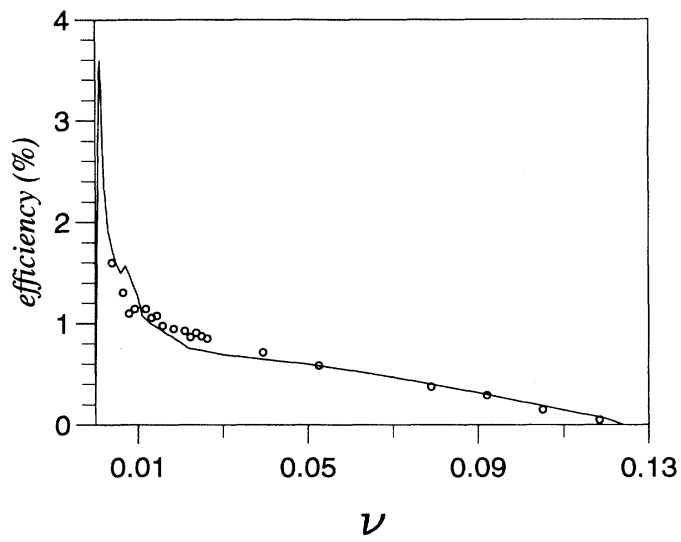

FIG. 17. Efficiency vs cavity detuning $\nu$ for $\alpha=0.02$; continuous line: average efficiency $\eta=\alpha \mathcal{E} / 4 \pi N_{w}$ as it results from the numerical integration of Eqs. (16)-(20); circles: experimental data for $\lambda=25 \mu \mathrm{m}$, at the end of the macropulse (a.u.) (measurements carried out on the FELIX experiment).

Limit-cycle behavior is observed in a range $0.017<\nu<$ 0.03 (corresponding to a range $0.6<\delta \mathcal{L} / \lambda<1$ ) $[27,30]$. Period doubling should be observable for a macropulse longer than $10 \mu s$, in the range $0.005<\nu<0.017$, as can be seen from Fig. 7 , with $\alpha \approx 0.015$. For $\nu>0.03$, a quasistable or damped operation is asymptotically reached.

When undergoing a limit cycle, two supermodes are excited in the nonlinear part of the evolution of the macropulse. As discussed above, a limit cycle is due to a coupling between the first and second supermodes. We find that the frequency is close to $\omega_{\mathrm{LC}} \approx(3 / 2)(\nu / 2)^{2 / 3}$ [see Eq. (56)], i.e., has a period of approximately $T \approx$ $(3 / 2)(\lambda / \delta \mathcal{L})^{2 / 3}(\mu \mathrm{s})$ for a cavity round-trip time of 40 ns. This value should be compared with the fit of the experimental data, $T \approx\left[(\lambda / \delta \mathcal{L})^{0.64}(\mu \mathrm{s})\right][33]$.

The macropulse oscillation frequency is related to the optical microstructure, which consists of a train of regularly spaced subpulses that are formed during the limit cycle, as discussed in Sec. VI. The separation between optical spikes in the substructure of a single micropulse, as in Fig. 14, has been measured, using second-order autocorrelation techniques, at $\lambda=24.5 \mu \mathrm{m}$, to be approximately $1.23 \Delta$ for $\delta \mathcal{L} \approx 0.65 \lambda$ [34]. This measured value should be compared with $1.7 \Delta$ calculated from our approximate expression for $T$.

In Fig. 18 we show some examples of $\lambda=40 \mu \mathrm{m}$ macropulse shapes for different $\delta \mathcal{L}$ values, and representing different regimes of operation: a quasistable operation (dotted line), with $\delta \mathcal{L}=1.55 \lambda(\nu=0.038)$, two cases of period-doubling behavior (long-dashed and dash-dotted lines), with $\delta \mathcal{L}=0.55 \lambda(\nu=0.0135)$ and $\delta \mathcal{L}=0.35 \lambda(\nu=0.0085)$, respectively, and two cases of chaotic behavior (dashed and continuous lines), with $\delta \mathcal{L}=0.15 \lambda(\nu=0.0034)$ and $\delta \mathcal{L}=0.1 \lambda(\nu=0.0024)$, respectively. In Fig. 18(a), showing the experimental data, the macropulse ends at $10.5 \mu \mathrm{s}$, after which the power decays exponentially due to cavity loss. The simulations show that period doubling and chaos should be observ- able for a longer macropulse. Our reduced model, however, only gives a qualitative description of the real behavior. A more accurate description can be obtained by solving the multielectrons Eqs. (11) and (12).

Superradiance should be observable in short electron bunch FEL's where the bunches are shorter than or of the order of the slippage length. Because the gain is small at the $\delta \mathcal{L}$ for optimum efficiency, where superradiance is observable, the time to saturation is usually longer than the macropulse. However, in FEL's driven by superconducting accelerators [23], superradiance may indeed be observable with a judicious choice of the cavity loss. For shorter macropulses, such as those of FELIX, the technique of dynamics desynchronism $[16,17,35]$ may shorten the buildup time to saturation by allowing the laser to start up with $\delta \mathcal{L}=\delta \mathcal{L}_{\text {opt }}$ for maximum gain and saturate with $\delta \mathcal{L}=0.1815 \gamma \Delta \alpha^{3 / 2}$ (see Sec. V), for maximum efficiency and superradiant operation. For small cavity loss, the already very short optical pulses gener-
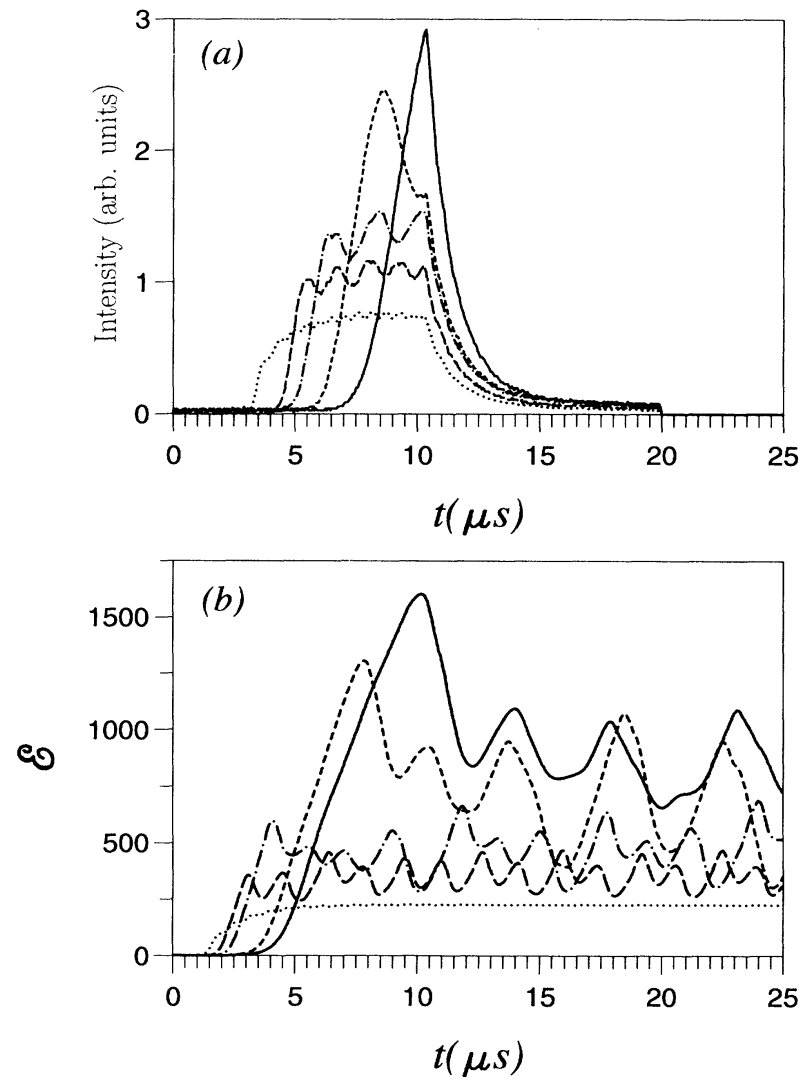

FIG. 18. (a) The measured [27] and (b) simulated macropulse shapes at $\lambda=40 \mu \mathrm{m}$ for different $\delta \mathcal{L}$ values, for the parameters of Table I, showing the various regimes of operation. Dotted line: quasistable operation, with $\delta \mathcal{L}=1.55 \lambda(\nu=0.038)$; long-dashed and dash-dotted lines: period-doubling behavior, with $\delta \mathcal{L}=0.55 \lambda(\nu=0.0135)$ and $\delta \mathcal{L}=0.35 \lambda(\nu=0.0085) ;$ dashed and continuous lines: chaotic behavior, with $\delta \mathcal{L}=0.15 \lambda(\nu=0.0034)$ and $\delta \mathcal{L}=0.1 \lambda(\nu=0.0024)$. 
ated by the short-bunch FEL (e.g., 6 period long pulses in FELIX [34]) can be further shortened to the limit of $N_{s}=0.56 N_{w} \sqrt{\alpha}$, by reducing $\alpha$.

\section{CONCLUSIONS}

In conclusion, we have studied the linear and nonlinear evolution of the radiation pulse in a small-gain FEL driven by electron bunches shorter than the slippage length and longer than the radiation wavelength. We have analytically calculated the eigenmodes of the linear regime (supermodes) as a function of the scaled cavity shortening parameter $\nu$. As $\nu$ decreases, the number of supermodes with positive gain increases. The linear gain, as measured in the FELIX experiment operating with electron pulses shorter than the slippage length, corresponds to the gain of the first supermode. From the linear analysis, we have shown that for very small cavity shortening several supermodes with comparable gain are linearly excited. The nonlinear regime has been investigated using a collective model using a closure relation [11], that reduces the multiparticle equations to a set of five nonlinear partial differential equations for the radiation field and the first moments of the electron distribution. In the single supermode approximation and for moderate values of saturation, the equations reduce to a Landau-Ginzburg equation, which allow one to obtain analytical expressions for the optical energy and nonlinear frequency shift at saturation as a function of the scaled cavity shortening $\nu$ and loss $\alpha$. From the approximate solution we have demonstrated that the radiation is superradiant at a cavity shortening corresponding to the optimum efficiency.
Explicit expressions for optimum peak power, efficiency, and pulse width have also been derived. In the second part of the paper multisupermode operation has been discussed, interpreting the limit-cycle and period-doubling behavior as a competition between different supermodes. We have numerically investigated the transition from the asymptotically stable regime, where the first supermode dominates, to a limit cycle, where two nonlinear coupled supermodes are excited to a comparable intensity level. The frequency of the limit cycle has been interpreted as the difference between the two lower supermode frequencies, $\omega_{L C}=\omega_{2}-\omega_{1}$. A further reduction of the cavity length shows the transition to a chaotic regime via a period doubling cascade. Finally, we have compared our theoretical treatment with experimental observations from the FELIX experiment and found good agreement.

Our theoretical approach also provides an understanding and an explantion of the narrow, high-intensity peak observed in the efficiency detuning curve of short bunch FEL's.

\section{ACKNOWLEDGMENTS}

Helpful discussions with R. Bonifacio, J.S. Wurtele, N.S. Ginzburg, and P.L. Lions are gratefully acknowledged. The FOM Instituut voor Plasmafysica "Rijnhuizen" in The Netherlands is acknowledged for the financial support of D.A.J for the duration of the experimental work and the support of the FELIX team during the experimental measurements is gratefully acknowledged.
[1] W.B. Colson and A. Renieri, J. Phys. (Paris) Colloq. 44, C1-11 (1983).

[2] J.M.J. Madey, Nuovo Cimento 50, 1 (1979).

[3] R. Bonifacio, B.W.J. McNeil, and P. Pierini, Phys. Rev. A 40, 4467 (1989).

[4] R. Bonifacio, C. Pellegrini, and L. Narducci, Opt. Commun. 50, 373 (1984).

[5] Y.A. Bogomolov, V.L. Bratman, N.S. Ginzburg, M.I. Petelin, and A.D. Yunakovsky, Opt. Commun. 36, 209 (1981).

[6] W.B. Colson, Phys. Quantum Electron 8457 (1982); in Laser Handbook, edited by W.B. Colson, C. Pellegrini, and A. Renieri (Elsevier, B.V., Amsterdam, 1990), Vol. 6, p. 115 .

[7] S.J. Hahn and J.K. Lee, Phys. Lett. A 176, 339 (1993); Phys. Rev. E 48, 2162 (1993).

[8] Y. Pinhasi and A. Gover, Nucl. Instrum. Methods Phys. Res., Sect. A 358, 86 (1995).

[9] N. Piovella, Phys. Rev. E 51, 5147 (1995).

[10] N.S. Ginzburg, S.P. Kuznetsov, and T.N. Fedosseva, Radio Phys. Quantum Electron 21, 728 (1978).

[11] R. Bonifacio, F. Casagrande and L. De Salvo Souza, Phys. Rev. A 33, 2836 (1986).

[12] G. Shvets and J.S. Wurtele, Phys. Plasmas 1, 157 (1994).
[13] G. Dattoli, A. Marino, and A. Renieri, Opt. Commun. 35, 407 (1980).

[14] G. Dattoli, A. Marino, A. Renieri, and F. Romanelli, IEEE J. Quantum Electron. 17, 1371 (1981).

[15] P. Ellaume, IEEE J. Quantum Electron. 21, 1012 (1985).

[16] D.A. Jaroszynski, D. Oepts, A.F.G. van der Meer, P.W. van Amersfoort, and W.B. Colson, Nucl. Instrum. Methods Phys. Res., Sect. A 296, 480 (1990).

[17] G.T. Moore and N. Piovella, IEEE J. Quantum Electron. 27, 2522 (1991).

[18] R. Bonifacio, R. Corsini, L. De Salvo Souza, P. Pierini, and N. Piovella, Rivista del Nuovo Cimento 15, 1 (1992).

[19] R. Bonifacio, L. De Salvo Souza, P. Pierini, and N. Piovella, Nucl. Instrum. Methods Phys. Res., Sect. A 296, 358 (1990).

[20] F. Hopf, P. Meystre, and D.W. McLaughin, Phys. Rev. A 13, 777 (1976).

[21] R.J. Bakker et al., J. Appl. Phys. 74, 1501 (1993); IEEE J. Quantum Electron. 30, 1635 (1994).

[22] F. Glotin et al., Phys. Rev. Lett. 71, 2587 (1993).

[23] W.P. Leemans et al., Nucl. Instrum. Methods Phys. Res., Sect. A 385, 208 (1995).

[24] D.A. Jaroszynski, D. Oepts, J.M. Ortega, C.R. Pidgeon, and P.W. van Amersfoort, Nucl. Instrum. Methods Phys. 
Res., Sect. A 318, 582 (1992).

[25] R. Bonifacio, L. De Salvo, P. Pierini, N. Piovella, and C. Pellegrini, Phys. Rev. Lett. 73, 70 (1994).

[26] D. Iracane, P. Chaix, and J.L. Ferrer, Phys. Rev. E 49, 800 (1994).

[27] D.A. Jaroszynski et al., Phys. Rev. Lett. 70, 3412 (1993).

[28] D.A. Jaroszynski et al., Phys. Rev. Lett. 71, 3798 (1993).

[29] H.T. Moon, P. Huerre, and L.G. Redekopp, Physica (Amsterdam) 7D, 135 (1983).

[30] D.A. Jaroszynski, R.J. Bakker, D. Oepts, A.F.G. van der Meer, and P.W. van Amersfoort, Nucl. Instrum. Methods
Phys. Res., Sect. A 331, 52 (1993).

[31] D.A. Jaroszynski et al., Phys. Rev. Lett. 74, 2224 (1995).

[32] W.A. Gillespie et al., Nucl. Instrum. Methods Phys. Res., Sec. A 358, 232 (1995).

[33] D. Oepts et al., FOM, FELIX Report No. 91-210 (1991) (unpublished).

[34] G.M.H. Knippels, R.F.X.A.M. Mols, A.F.G. van der Meer. D. Oepts, and P.W. van Amersfoort, Phys. Rev. Lett. 75, 1755 (1995).

[35] R.J. Bakker et al., Phys. Rev. E 48, 3256 (1993). 